\title{
A tessellated continuum approach for the static analysis of perforated structures
}

DOI:

10.1016/j.compstruc.2019.106140

\section{Document Version}

Accepted author manuscript

Link to publication record in Manchester Research Explorer

\section{Citation for published version (APA):}

Davey, K., Darvizeh, R., \& Sedqi, Z. (2020). A tessellated continuum approach for the static analysis of perforated structures. Computers and Structures, 227, [106140]. https://doi.org/10.1016/j.compstruc.2019.106140

\section{Published in:}

Computers and Structures

\section{Citing this paper}

Please note that where the full-text provided on Manchester Research Explorer is the Author Accepted Manuscript or Proof version this may differ from the final Published version. If citing, it is advised that you check and use the publisher's definitive version.

\section{General rights}

Copyright and moral rights for the publications made accessible in the Research Explorer are retained by the authors and/or other copyright owners and it is a condition of accessing publications that users recognise and abide by the legal requirements associated with these rights.

\section{Takedown policy}

If you believe that this document breaches copyright please refer to the University of Manchester's Takedown Procedures [http://man.ac.uk/04Y6Bo] or contact uml.scholarlycommunications@manchester.ac.uk providing relevant details, so we can investigate your claim.

\section{OPEN ACCESS}




\title{
A Tessellated Continuum Approach for the Static Analysis of Perforated Structures
}

\author{
K. Davey ${ }^{a, b}$, R. Darvizeh ${ }^{a}$, Z. Sedqi ${ }^{a}$ \\ ${ }^{a}$ School of Mechanical, Aerospace and Civil Engineering, The University of Manchester \\ ${ }^{b}$ The Advanced Forming Research Centre, The University of Strathclyde
}

\begin{abstract}
Pre-fractals have the capacity to represent structures of different levels of complexity and have recently been shown to be suitable for continuum analysis using a method called tessellated continuum mechanics. Tessellated continuum mechanics is an analytical theory for the analysis of porous/perforated structures but presently has only been tested to any extent on heat transfer problems.

This paper is concerned with extending the implementation of the tessellated approach to static structural analysis of one and two dimensions with a particular emphasis on perforated plates. The principal objective of the work is to establish that the structural analysis of perforated structures is possible to a very high degree of accuracy in a continuum mechanics framework. The tessellated approach involves the local expansion of space to close perforations and invokes the concept of finite similitude, which has appeared in the recent literature.
\end{abstract}

The consequences of local-space scaling are examined and static testing for beams and plates, constrained by different boundary conditions are presented.

Keywords: static analysis; finite similitude; perforated plates; pre-fractals; numerical solutions. 


\section{Introduction}

Mandelbrot [1] popularised a different form of complex geometry that hitherto was considered a mathematical curiosity being excluded from the Euclidean definition of geometry and considered "formless". These geometries are known as fractal. Although interest in fractals has increased following Mandelbrot's work [2] their application to physical systems remains at best problematic. Practical application of fractals remains somewhat limited and references [3-9] provide a snapshot of what is evidently far from mainstream use. The difficulty with fractals stems from an inability to quantify traditional measures of volume, area and length and consequently, traditional mechanics is not possible. Fractals provide a precise definition of geometry, which can be conveniently constructed by a convergent sequence of pre-fractals but they do not exist in reality. Prefractals, however, retain traditional measures of volume, area and length and can themselves provide excellent representations of physical holey artefacts. Pre-fractals also benefit from the very efficient construction procedures associated with fractals, i.e. recursion and iterated function schemes [10].

Although pre-fractals provide an efficient means of representing perforated structures, their application in solid mechanics is presently limited. Some of the few studies appearing in the open literature are the loading of a von Koch beam structure [11] and the dynamic response of a Pascal-Sierpinski Gasket in dynamical analysis [12]. The static and dynamic response of holey plates, however, is extensive as these types of structures are found in a range of practical applications. The reader is directed to the expanded literature review on implementing perforated plates in engineering applications by Lewinski [13]. Some of the engineering uses of perforated plate are: as barriers to shock waves, absorbers, separation planes for fluids and solids like soils [14-17]. The analysis in this paper is restricted to electrostatic behaviour and consequently, a constitutive law for elasticity is essential to generate general equations of motion. For plates with holes, a particular consideration is the thickness of the plate $h$ compared to the smallest distance between the neighbouring 
holes $\varepsilon$, where the inequality $h<<\varepsilon[13]$ is advantageous in order to avoid the need for the application of full-plate theory in place of the more convenient membrane-plate theory. Kalamkarov et al. [18], investigated the issues for the case where $\varepsilon$ and $h$ are of similar size and developed a general asymptotic homogenous composite shell model to deal with the situation; subsequently used in numerous applications by many researchers [18]. Despite the simplifying benefits of asymptotic or membrane theory, the computational analysis of perforated structures offers particular challenges, which has led many researchers to seek further simplifications. One approach to account for the effect of holes is to neglect them explicitly and incorporate them implicitly by means of effective properties in the governing equations. Needless to say, such approaches offer only low resolution and are not appropriate for high-accuracy applications. Pan'kov et al. [19] for example used an average field-theory approach to approximate the bending properties of composites involving uniform perforations but punctuated with high-density non-uniform holes. Overwhelmingly the vast majority of analysis approaches for perforated plates are approximate.

The work proposed in this paper is founded on tessellated continuum mechanics (TCM), which is designed to relate pre-fractal responses to those on a tessellation. Both the prefractals and tessellations are created using iterated function schemes (IFS). Both involve the exact same number of contraction maps, which are used to form pre-fractals and tessellations but no holes are formed when constructing tessellations. The tessellated approach aims to lift solutions from a continuum and map them to a pre-fractal. In this way, classical theories in continuum mechanics can be applied. See for example the work in reference [20] concerned with the determination of analytical solutions for heat-transfer problems on porous structures represented by pre-fractals and fractals. Tessellations and pre-fractals are related by means of a hole-fill map [21], which as the name suggests has the effect of closing all the holes in pre-fractal to produce a tessellation. A particular difficulty with hole closing is that discontinuous fields can appear on a tessellation, which can be 
accounted for however with a discontinuity network [22]. Although the tessellated approach has been developed numerically with a Galerkin finite element code [23] its application is presently limited to heat transfer problems.

In parallel with the development of the tessellated theory, a related activity concerned with scaling has appeared in the open literature. The scaling work is founded on the idea that scaling can be achieved through the expansion/contraction of space itself. The first application of the concept was to metal-forming processes [24], [25]. More recently the same method has been validated in bio-mechanical experimentation under isotropic scaling [26] and in the scaling of the powder compaction process [27]. Although the scaling work reported only applies to isotropic scaling it potentially can be applied to anisotropic scaling and to local regions of space. The link between anisotropic space scaling and tessellated continuum mechanics is established in this paper.

The particular focus of the paper is on demonstrating how tessellated continuum mechanics can be applied to static applications. This is a step-up from previously analysed scalar-field heat-transfer problems as deformation involves vector fields. Pre-fractals for classical fractals are considered, i.e. the Cantor Set (embedded in a 1-D space) and the Sierpinski Carpet and the Vicsek fractal (embedded in a 2-D space) [10]. A range of geometries, boundary conditions and loading sources are considered to demonstrate that all types of static problem can be accommodated by the theory.

Introduced in Section 2 is the basic terminology and the recursive schemes for the construction of both pre-fractals and tessellations and the associated hole-file maps. The concept of finite similitude, which arises out of scaling theory, is introduced in Section 3. In Sections 4, 5 and 6 it is demonstrated how physics coupling across two spaces is achieved taking into consideration mechanical definitions, constitutive behaviour and moments. To illustrate how the new tessellated theory is applied in practice, procedural steps are presented in Section 7. Case studies are presented in Section 8 and 9 for beams 
and plates, where the high precision of the tessellated approach is confirmed. The paper ends with conclusions.

\section{Foundational concepts}

This section is concerned with foundational concepts for the application of tessellated continuum mechanics, which includes the recursive approach to create pre-fractals and tessellation along with hole-fill maps, which connects elements in pre-fractals and tessellations in a one-to-one relationship. Discontinuity networks are also examined along with the type of jump conditions that occur in practice.

\subsection{Fractals, Pre-fractals and Tessellations}

As mentioned above fractals are 'formless' shapes that are considered outside the traditional shapes common to Euclidean geometry [1]. Fractals are typically defined in a limiting process and in the limit, measures of volume, area and length can be undefined being infinite or zero. Although it is possible to define alternative finite measures of "size" on a fractal these bear little relationship to physical quantities met in mechanics. Fractals have the advantage of being precisely defined by a limiting process, which is not the case for pre-fractals. However, pre-fractals approximate more meaningfully physical features in nature as traditional measures remain finite since no limit is taken. This property along with the fact that pre-fractals can be constructed efficiently and offer high complexity are the reasons for focusing on them here.

Pre-fractals can be constructed by using an iterated function system (IFS), which are particularly convenient for the creation of self-similar structures [27]. Self-similarity is a feature common to many porous and perforated structures that appear in nature although its manifestation tends to be restricted to a limited range of space (sometimes time) scales [10]. To illustrate the process consider the construction of pre-fractals in a 1-D Cantor Set, which 
involves two contraction maps, i.e. $S_{1}=x / 3$ and $S_{2}=\left(2 \ell_{0}+x\right) / 3$. Figure 1 (a) shows the effect of applying these maps in a recursive manner with the generation of pre-fractals $\hat{E}_{k}$, $k=0,1 \ldots$. Depicted in the figure are $\hat{E}_{0}=\left[0, \ell_{0}\right], \hat{E}_{1}=S_{1}\left(\hat{E}_{0}\right) \cup S_{2}\left(\hat{E}_{0}\right), \hat{E}_{2}=S_{1}\left(\hat{E}_{1}\right) \cup S_{2}\left(\hat{E}_{1}\right)$ which leads to the general recursive relationship $\hat{E}_{k}=S_{1}\left(\hat{E}_{k-1}\right) \cup S_{2}\left(\hat{E}_{k-1}\right)$. The process to create tessellations parallels the pre-fractal construction process with the principal difference being the maps applied are selected to avoid holes appearing in the continuum. The tessellations depicted in Figure 1(b) are created using the maps $P_{1}=x / 2$ and $P_{2}=\left(\ell_{0}+x\right) / 2$, where $\hat{T}_{0}=\left[0, \ell_{0}\right], \hat{T}_{1}=P_{1}\left(\hat{T}_{0}\right) \cup P_{2}\left(\hat{T}_{0}\right), \hat{T}_{2}=P_{1}\left(\hat{T}_{1}\right) \cup P_{2}\left(\hat{T}_{1}\right)$, which again leads to the general recursive relationship $\hat{T}_{k}=P_{1}\left(\hat{T}_{k-1}\right) \cup P_{2}\left(\hat{T}_{k-1}\right)$. A peculiarity of the tessellation is that (when considered as a set of points) $\hat{T}_{k}=\hat{T}_{0}$, i.e. the continuum remains unchanged. Note however that $\hat{T}_{k}$ is formed by the union of $2^{k}$ closed intervals, which is the exact same number of closed intervals to form $\hat{E}_{k}$. The corresponding closed intervals in $\hat{T}_{k}$ and $\hat{E}_{k}$ can be placed in direct correspondence with each other through what is termed a "hole-fill" map. Prior to considering hole-fill maps however it of interest to see how the construction procedure for pre-fractals and tessellations work in 2D. For example, to construct the pre-fractal in a 2-D Sierpinski Carpet, eight types of contraction maps are needed to create the pre-fractal $\hat{E}_{k}$, which are of the form $\boldsymbol{S}_{k}=A_{k} \cdot \boldsymbol{x}+\boldsymbol{c}_{k}$, where $\boldsymbol{x}=(x, y)$ and $A_{1}=\cdots=A_{8}=\frac{1}{3}\left[\begin{array}{ll}1 & 0 \\ 0 & 1\end{array}\right]$

and where $\boldsymbol{c}_{2}=(0,0), \boldsymbol{c}_{2}=\left(\ell \quad, \boldsymbol{c}_{3}=\left(2 \ell \quad, \boldsymbol{c}_{4}=\left(0, \ell \quad, \boldsymbol{c}_{5}=(2 \ell \quad \ell \quad\right.\right.\right.$,

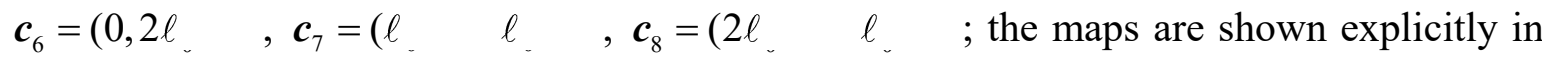


Appendix A1.

Shown in Figure 2(a) is $\hat{E}_{1}$, which is obtained from the union of these eight maps applied to the square $\hat{E}_{0}=\left[0, \ell_{0}\right] \times\left[0, \ell_{0}\right]$, i.e. $\hat{E}_{1}=\bigcup_{i=1}^{8} S_{i}\left(\hat{E}_{0}\right)$. Note also that $\hat{E}_{0}$ is endowed with an initial tessellation which is transferred to $\hat{E}_{1}$ in Figure 2(a) and is further transferred to $\hat{E}_{2}=\bigcup_{i=1}^{8} S_{i}\left(\hat{E}_{1}\right)$ in Figure 2(c). The initial tessellation in these figures is required to aid in the construction of tessellations $\hat{T}_{k}$, with $\hat{T}_{1}$ shown in Figure 2(b) and $\hat{T}_{2}$ depicted in Figure 2(d). Shown in Figure 2 are arrows showing the association between corresponding elements in the $\hat{E}_{1}$ and $\hat{T}_{1}$. The tessellations are formed with the recursive relationship $\hat{T}_{k}=\bigcup \quad$, which matches that for the pre-fractals, i.e. $\hat{E}_{k}=\bigcup_{i=1}^{8} S_{i}\left(\hat{E}_{k-1}\right)$ with the only difference being the contraction maps used. The contraction maps $P_{i}$ are employed for the tessellation instead of $S_{i}$, where $P_{i}$ are provided in Appendix A2. The number of maps involved in both constructions is identical (equal to eight) but $P_{i}$ is formed by a number of sub-maps and this is the purpose of the initial tessellation on $\hat{T}_{0}$. The submaps that form $P_{i}$ are selected to close the holes in $\hat{E}_{1}$ as illustrated in Figure 2. The initial tessellation on $\hat{T}_{0}$ consists of eight triangular elements which means that $P_{i}$ is formed by eight linear sub-maps. It is this feature that enables tessellations to be created recursively without the appearance of holes and match exactly the number of elements in the corresponding pre-fractals.

\subsection{Hole-fill maps}

Although the maps chosen to form a tessellation have regard for the maps involved in forming a pre-fractal (as discussed above) the generation procedures for both are essentially independent. It is necessary therefore to define a map that relates points in a pre-fractal to 
those on a tessellation. The map involved is termed a "hole-fill map" since it has the appearance of closing holes on a pre-fractal if applied to it. Hole-fill maps are not unique since holes in a pre-fractal can be closed in a variety of ways depending on the initial tessellation on $\hat{T}_{0}$ and hence the maps $P_{i}$. It is important that this lack of uniqueness however does not influence the solution to any physical problem defined on a pre-fractal. Two approaches exist for the creation of hole-fill maps one suitable for analytical work and the other best suited to numerical analysis.

\subsubsection{Function Composition}

Since recursion is used to form both tessellations and pre-fractals, starting from identical initial domains $\hat{T}_{0}=\hat{E}_{0}$, it follows that a point $\boldsymbol{x} \in \hat{T}_{0}=\hat{E}_{0}$ is mapped to $\hat{E}_{k}$ and $\hat{T}_{k}$ through corresponding function compositions. Thus, for example, $\boldsymbol{y} \in \hat{E}_{k}$ is related to $\boldsymbol{x} \in \hat{E}_{0}$ through the $k$ compositions $S_{i} \circ S_{j} \circ \cdots \circ S_{k}$, i.e. $\boldsymbol{y}=S_{i} \circ S_{j} \circ \cdots \circ S_{k}(\boldsymbol{x})$ and similarly $\boldsymbol{z} \in \hat{T}_{k}$ is related to $\boldsymbol{x} \in \hat{T}_{0}$ through the corresponding $k$ compositions $P_{i} \circ P_{j} \circ \cdots \circ P_{k}$ to give $\boldsymbol{z}=P_{i} \circ P_{j} \circ \cdots \circ P_{k}(\boldsymbol{x})$. The inverse map that takes the point $\boldsymbol{y} \in \hat{E}_{k}$ to $\boldsymbol{x} \in \hat{E}_{0}$ is $S_{k}^{-1} \circ \cdots \circ S_{j}^{-1} \circ S_{i}^{-1}$ with $\boldsymbol{x}=S_{k}^{-1} \circ \cdots \circ S_{j}^{-1} \circ S_{i}^{-1}(\boldsymbol{y})$. It follows immediately that $\boldsymbol{y} \in \hat{E}_{k}$ is related to $z \in \hat{T}_{k}$ through the hole-fill map $P_{i} \circ P_{j} \circ \cdots \circ P_{k} \circ S_{k}^{-1} \circ \cdots \circ S_{j}^{-1} \circ S_{i}^{-1}$ since $P_{i} \circ P_{j} \circ \cdots \circ P_{k} \circ S_{k}^{-1} \circ \cdots \circ S_{j}^{-1} \circ S_{i}^{-1}(\boldsymbol{y})=P_{i} \circ P_{j} \circ \cdots \circ P_{k}(\boldsymbol{x})=\boldsymbol{z}$

Forming the hole-fill map $P_{i} \circ P_{j} \circ \cdots \circ P_{k} \circ S_{k}^{-1} \circ \cdots \circ S_{j}^{-1} \circ S_{i}^{-1}$ symbolically is rather cumbersome and best suited to analytical work. The indexing associated with sub-maps is not included here for clarity sake.

\subsubsection{Direct formulation}


The alternative approach to relating points in $\hat{E}_{k}$ to $\hat{T}_{k}$ is to do this directly through corresponding triangular elements. The association between triangular elements in $\hat{E}_{k}$ and $\hat{T}_{k}$ are known by the fact that the recursive procedure forming these is identical. A selection of corresponding elements is highlighted in Figure 2. A particular advantage of using triangular elements is that the associated map is linear and takes the form

$\boldsymbol{z}=F_{i, j, \ldots, k}(\boldsymbol{y})+\boldsymbol{c}_{i, j, \ldots, k}$

which is, in fact, identical to Equation (2), where again the indexing associated with submaps is purposely absent here. The direct formulation is ideally suited to numerical work as the linear maps between triangular elements are readily handled numerically.

\subsection{Discontinuity Network}

The closing of holes in a pre-fractal to form a tessellation can result in a jump in a field variable on the tessellation. For example displacement of either side of a hole need not be identical and on a tessellation absent of holes this would appear as a jump discontinuity. It is necessary therefore to be able to identify where discontinuities occur in a tessellation and the collection of these locations are termed a discontinuity network [23]. In forming tessellations the location of discontinuities on a discontinuity are readily identified as part of the recursive process.

\section{Finite similitude theoretical background}

The concept of finite similitude has appeared in the recent literature [24] and is concerned with the matching of continuum physics in two spaces. With this approach one of the spaces (the trial space) can be viewed as a distorted version of the physical space making it particularly useful for scaled experimentation. Finite similitude applies to the whole region of space, so is not immediately transferable to tessellated continuum 
mechanics. However, the existence of a hole-fill map raises the possibility that the physics on pre-fractals and tessellations can be related through the mechanism of local-space distortion. Each element in a pre-fractal is related in a one-to-one correspondent to an element in a tessellation (see Section 2) and therefore the physics between the two corresponding elements can in principle be matched locally according to the rules of finite similitude theory. The theory requires that the space-distortion map is an affine map and indeed this is the case here for the hole-fill map as confirmed by Equation (3). Since finite similitude is concerned with regions of space it naturally gives rise to the control volume concept. Finite similitude for tessellated continuum mechanics is therefore founded on the idea of proportional transport equations representing the governing physics in pre-fractal and tessellated elements. The transport form of the governing equations is suitable also for representing the discontinuous physics pervasive to pre-fractal and tessellated geometries and for the capture of all conservative and non-conservative physical laws. The focus in this paper being on the static behaviour of continuum mechanical structures can be described by equations of the type:

$-\int_{\Gamma_{i}^{*}} \underline{J}_{i}^{\psi} \cdot \underline{n}_{i} d \Gamma_{i}^{*}+\int_{\Omega_{i}^{*}} \rho_{i} b_{i}^{\psi} d V_{i}^{*}=0$

where $\Omega^{*}$ is a control volume (CV) in the form of a continuous open set of points surrounded by an orientable boundary $\Gamma^{*}$ and the subscript $i$ refers to the $\mathrm{i}^{\text {th }}$ element (either pre-fractal or tessellation). Furthermore, $\psi$ is a field variable, $\rho$ is material density, $\underline{n}$ is a unit normal vector pointing outward, $b$ is a source term and $\underline{J} \cdot \underline{n}$ is a flux.

Equation (4) is a general form that can be used to capture the conservative and nonconservative quasi-static processes, which are assumed to be applicable on both spaces. It is convenient to drop the subscript $i$ from Equation (4) at this point and relate two arbitrary corresponding elements in the pre-fractal and tessellation by the differential $\boldsymbol{d} \boldsymbol{x}_{t s}=F \cdot \boldsymbol{d} \boldsymbol{x}_{p s}$. Here the subscripts $p s$ and $t s$ denote pre-fractal and tessellated spaces, respectively and $F$ is 
the hole-fill map described in Section 2 (see Equation (3)) with all subscripts dropped for convenience and clarity. The relationship $\boldsymbol{d} \boldsymbol{x}_{t s}=F \cdot \boldsymbol{d} \boldsymbol{x}_{p s}$ enables Nanson's identities [29] to be applied to related incremental volumes and areas, i.e. $d V_{t s}=J d V_{p s}$ and $\underline{d \Gamma}_{t s}=J F^{-T} \cdot \underline{d \Gamma_{p s}}$, where $J=\operatorname{det} F, \underline{d \Gamma_{t s}}=\underline{n}_{t s} d \Gamma_{t s}$ and $\underline{d \Gamma_{p s}}=\underline{n}_{p s} d \Gamma_{p s} . \quad$ Armed with these identities Equation (4) provides three equations, i.e.

$$
\begin{aligned}
& -\int_{\Gamma_{p s}^{*}} \underline{J}_{p s}^{\psi} \cdot \underline{n}_{p s} d \Gamma_{p s}^{*}+\int_{\Omega_{p s}^{*}} \rho_{p s} b_{p s}^{\psi} d V_{p s}^{*}=0 \\
& -\int_{\Gamma_{\Gamma_{s}^{*}}^{*}} \underline{J}_{t s}^{\psi} \cdot \underline{n}_{t s} d \Gamma_{t s}^{*}+\int_{\Omega_{s s}^{*}} \rho_{t s} b_{t s}^{\psi} d V_{t s}^{*}=0 \\
& -\int_{\Gamma_{p s}^{*}}\left(F^{-1} \cdot \underline{J}_{t s}^{\psi}\right) \cdot \underline{n}_{p s} d \Gamma_{p s}^{*}+\int_{\Omega_{p s}^{*}} \rho_{t s} b_{t s}^{\psi} J d V_{p s}^{*}=0
\end{aligned}
$$

where on multiplication of the last equation with $\alpha^{\psi}>0$ (since seeking proportional physics) or $\alpha^{\psi} G^{\psi}$ (for a vector equation) and contrasting with the first provides the general scaling relationships

$\rho_{p s} b_{p s}^{\psi}=\alpha^{\psi} \rho_{t s} b_{t s}^{\psi} J$

and

$\underline{J}_{p s}^{\prime \prime}=\alpha^{\psi} J F^{-1} \cdot \underline{J}_{t s}^{\prime \prime}$

or alternatively for vector equations

$$
\rho_{p s} \underline{b}_{p s}^{\psi}=\alpha^{\psi} \rho_{t s} J G^{\psi} \cdot \underline{b}_{t s}^{\psi}
$$

and

$$
\underline{\underline{J}}_{p s}^{\psi}=\alpha^{\psi} J G^{\psi} \cdot J_{t s}^{\psi} \cdot F^{-T}
$$

where $G^{\psi}$ is an invertible scaling matrix. 
It should be noted that non-degeneracy of the inner product facilitates the absence of the unit normal $\underline{n}_{p s}$ from Equations (6b) and (7b) (since $\underline{n}_{p s}$ can point in any direction) and symmetry of the tensor $\underline{\underline{j}}_{p s}^{\psi}$ (and $\underline{\underline{J}}_{t s}^{\psi}$ ) immediately infers that $G^{\psi}=F^{-1}$ with scaling accounted for by $\alpha^{\psi}$ in Equation (7b).

\section{Mechanical relationships}

This section is concerned with the practical implementation of the general scaling equation theory presented in the previous subsection. This involves the application of particular equations pertinent to elastostatic problems. Consider first mass conservation, which in the two spaces means that $M_{p s}$ and $M_{t s}$ are constant where

$$
M_{p s}=\int_{\Omega_{p s}^{*}} \rho_{p s} d V_{p s}^{*} \text { and } M_{t s}=\int_{\Omega_{l s}^{*}} \rho_{t s} d V_{t s}^{*}
$$

which on the substitution of Nanson the identity $d V_{t s}^{*}=J d V_{p s}^{*}$ and multiplication by a scalar $\alpha^{\rho}>0$ (giving $M_{p s}=\alpha^{\rho} M_{t s}$ ), provides the relationship

$\rho_{p s}=\alpha^{\rho} J \rho_{t s}$

which ensures mass in the two spaces is proportional, where $\alpha^{\rho}$ is called the density scaling factor.

Consider further momentum conservation which being a vector equation means that Equation (7) is applicable, which provides the stress tensor identity

$$
\underline{\underline{\sigma}}_{p s}=\alpha^{v} J F^{-1} \cdot \underline{\underline{\sigma}}_{t s} \cdot F^{-T}
$$

where, as mentioned above, because of symmetric considerations $G^{v}=F^{-1}$, and body forces are related by $\rho_{p s} \underline{b}_{p s}=\alpha^{v} \rho_{t s} J F^{-1} \cdot \underline{b}_{t s}^{\psi}$ or in view of Equation (9) this reduces to 
$\alpha^{\rho} \underline{b}_{p s}=\alpha^{v} F^{-1} \cdot \underline{b}_{t s}^{\psi}$.

Consider further movement (defined by Davey and Darvizeh [24]), which in the two spaces means that $\underline{M}_{p s}$ and $\underline{M}_{t s}$ are constant where

$$
\underline{M}_{p s}=\int_{\Omega_{p s}^{*}} \rho_{p s} \underline{u}_{p s} d V_{p s}^{*} \text { and } \underline{M}_{t s}=\int_{\Omega_{l s}^{*}} \rho_{t s} \underline{u}_{t s} d V_{t s}^{*}
$$

which on the substitution of Nanson the identity $d V_{t s}^{*}=J d V_{p s}^{*}$ and multiplication by scalar $\alpha^{u} F^{-1}$ (giving $\underline{M}_{p s}=\alpha^{u} F^{-1} \underline{M}_{t s}$ ), provides the relationship

$\rho_{p s} \underline{u}_{p s}=\alpha^{u} \rho_{t s} J F^{-1} \cdot \underline{u}_{t s}$

but in view of Equation (9) reduces to $\alpha^{\rho} \underline{u}_{p s}=\alpha^{u} F^{-1} \cdot \underline{u}_{t s}$, where the choice $G^{u}=F^{-1}$ follows from the relationship $\boldsymbol{d} \boldsymbol{x}_{p s}=F^{-1} \cdot \boldsymbol{d} \boldsymbol{x}_{t s}$.

The final equation of interest is energy and for elastostatics, strain energy is of principal interest and in a control volume setting in the two spaces means that $E_{p s}$ and $E_{t s}$ are constant where

$$
E_{p s}=\frac{1}{2} \int_{\Omega_{p s}^{*}} \underline{\sigma}_{p s}: \underline{\varepsilon}_{p s} d V_{p s}^{*} \text { and } E_{t s}=\frac{1}{2} \int_{\Omega_{l s}^{*}} \underline{\sigma}_{t s}: \underline{\varepsilon}_{t s} d V_{t s}^{*}
$$

where $\underline{\underline{\varepsilon}}$ is a strain tensor and again on the substitution of Nanson the identity $d V_{t s}^{*}=J d V_{p s}^{*}$ and multiplication by a scalar $\alpha^{e}>0$ (giving $E_{p s}=\alpha^{e} E_{t s}$ ), provides the relationship

$$
\underline{\underline{\sigma}}_{p s}: \underline{\underline{\varepsilon}}_{p s}=\alpha^{e} J \underline{\underline{\sigma}}_{t s}: \underline{\underline{\varepsilon}}_{t s}
$$

Thus, relationships between the tessellated and fractal spaces have been established for density, stress, displacement, force and strain energy but in order to form a closed system of equations, a constitutive law is required relating stress to strain. 


\section{Strain and constitutive relationships}

The work here is limited to small deflection theory, so strain in the pre-fractal and tessellated spaces is the symmetric part of $\left(\partial u_{i} / \partial x^{j}\right)_{p s}$ and $\left(\partial u_{i} / \partial x^{j}\right)_{t s}$, respectively. A certain degree of care is required with regard to the position of subscripts and superscripts as distortion of space can lead to non-orthogonal coordinate systems. Therefore, in line with convention, upper scripts (superscripts) are termed contravariant with lower scripts (subscripts) being covariant. The coordinate functions, displacement vectors and stress tensors introduced in Section 4 are all contravariant. Thus, in suffix terms $\boldsymbol{d} \boldsymbol{x}_{t s}=F \cdot \boldsymbol{d} \boldsymbol{x}_{p s}$, $\alpha^{v} J \underline{\underline{\sigma}}_{t s}=F \cdot \underline{\underline{\sigma}}_{p s} \cdot F^{T} \quad$ and $\quad \alpha^{u} \underline{u}_{t s}=\alpha^{\rho} F \cdot \underline{u}_{p s} \quad$ are $\quad d x_{t s}^{i}=F^{i}{ }_{j} d x_{p s}^{j}, \quad \alpha^{v} J \sigma_{t s}^{i j}=F_{m}^{i} \sigma_{p s}^{m \ell} F_{\ell}^{j} \quad$ and $\alpha^{u} u_{t s}^{i}=\alpha^{\rho} F_{j}^{i} u_{p s}^{j}$, respectively. Strain however (i.e. $\left(\varepsilon_{i j}\right)_{p s}$ and $\left.\left(\varepsilon_{i j}\right)_{t s}\right)$ is required to be conjugate to stress and consequently must be covariant (since stress is contravariant) and hence the reason for the subscripts but note also that this requires the use of a covariant component of displacement with a subscript. Note further that the derivatives $\partial / \partial x_{p s}^{j}$ and $\partial / \partial x_{t s}^{j}$ are covariant and are related via the chain rule $\partial / \partial x_{p s}^{j}=F^{i}{ }_{j} \partial / \partial x_{t s}^{i}$ in view of the expression $d x_{t s}^{i}=F^{i}{ }_{j} d x_{p s}^{j}$. It is can be readily be deduced from $\alpha^{u} u_{t s}^{i}=\alpha^{\rho} F^{i}{ }_{j} u_{p s}^{j}$ that similarly convector coefficients for displacement are related via $\alpha^{\rho} u_{i p s}=\alpha^{u} \beta^{-1} F^{j}{ }_{i} u_{j t s}$, where $\beta^{3}=J$. To show this consider first the application of the chain rule $\partial / \partial x_{p s}^{j}=F_{j}^{i} \partial / \partial x_{t s}^{i}$, which gives $\alpha^{\rho} \partial u_{i p s} / \partial x_{p s}^{j}=\alpha^{u} \beta^{-1} F^{m}{ }_{j} F^{k}{ }_{i} \partial u_{k t s} / \partial x_{t s}^{m}$. Note further that Equation (14) in suffix notation gives [29]

$\underline{\underline{\sigma}}_{p s}: \underline{\varepsilon}_{p s}=\left(\sigma^{i j} \varepsilon_{i j}\right)_{p s}=\sigma_{p s}^{i j} \frac{\partial u_{i p s}}{\partial x_{p s}^{j}}=\alpha^{e} J \underline{\underline{\sigma}}_{t s}: \underline{\varepsilon}_{t s}=\alpha^{e} J\left(\sigma^{i j} \varepsilon_{i j}\right)_{t s}=\alpha^{e} J \sigma_{t s}^{i j} \frac{\partial u_{i t s}}{\partial x_{t s}^{j}}$

which on substitution of $\sigma_{p s}^{i j}=\alpha^{v} J F^{-1 i}{ }_{m}^{m \ell} \sigma_{t s} F_{\ell}^{-1 j}$ and $\alpha^{\rho} \partial u_{i p s} / \partial x_{p s}^{j}=\alpha^{u} \beta^{-1} F^{m}{ }_{j} F^{k}{ }_{i} \partial u_{k t s} / \partial x_{t s}^{m}$ 
into the left hand side gives

$$
\begin{aligned}
& \sigma_{p s}^{i j} \frac{\partial u_{i p s}}{\partial x_{p s}^{j}}=\left(\alpha^{\rho}\right)^{-1} \alpha^{v} \alpha^{u} \beta^{-1} J\left(F_{m}^{-1 i} \sigma_{t s}^{m \ell}{ }^{\prime} \quad \ell_{!} \bigcup_{{ }_{j}} F_{i} F_{i}^{k} \frac{\partial u_{k t s}}{\partial x_{t s}^{n}}\right) \\
& =\left(\alpha^{\rho}\right)^{-1} \alpha^{v} \alpha^{u} \beta^{-1} J\left(F_{i}^{k} F_{m}^{-1 i}\right)\left(F_{j}^{n} F^{-1 j}{ }_{\ell}\right) \sim^{m \ell} \frac{\partial_{u}}{\partial x_{t s}^{n}} \\
& =\left(\alpha^{\rho}\right)^{-1} \alpha^{v} \alpha^{u} \beta^{-1} J\left(\delta_{m}^{k}\right)\left(\delta_{\ell}^{n}\right) \tau^{m \ell} \frac{\partial u}{\partial x_{t s}^{n}}-j u ; \dot{j} u \mu \nu v_{t s}^{\ell} \frac{\partial u}{\partial x_{t s}^{\ell}}
\end{aligned}
$$

which matches the right-hand side of Equation (15) on setting $\alpha^{e}=\left(\alpha^{\rho} \beta\right)^{-1} \alpha^{v} \alpha^{u}$.

\subsection{Constitutive relationships in both spaces}

The scaling relationships considered thus far make no recourse to constitutive laws (although Equations (10) and (14) provide some constraint) and also only connect a single triangular tile in a tessellation to a corresponding triangular element in the pre-fractal. To proceed further, it is necessary to find a general elasticity relationship that applies in the tessellated space. The relationship between stress and strain in the physical space is

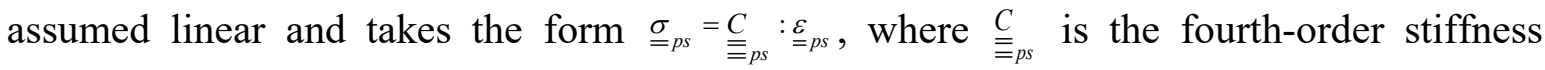

tensor. In suffix notation, this relationship is of the form $\sigma_{p s}^{i j}=C_{p s}^{i j k m} \varepsilon_{p s k m}$, where for an isotropic material (see reference [31]),

$$
C_{p s}^{i j k m}=K g_{p s}^{i j} g_{p s}^{k m}+\mu\left(g_{p s}^{i k} g_{p s}^{j m}+g_{p s}^{i m} g_{p s}^{j k}-\frac{2}{3} g_{p s}^{i j} g_{p s}^{k m}\right)
$$

where $K$ and $\mu$ are the bulk and shear modulus, which in terms of Poisson's ratio $v$, Young's modulus $E$ are related by the identities $3 K=E /(1-2 v)$ and $2 \mu=E /(1+v)$.

The symbol $g_{p s}^{i j}$ appearing in Equation (17) is a $2^{\text {nd }}$-order metric tensor [31], which for 
an orthogonal coordinate system equates to the Kronecker delta symbol $\delta^{i j}$. The metric tensor is symmetric (i.e. $g_{p s}^{i j}=g_{p s}^{j i}$ ) and it can be seen on inspection of Equation (17) that the stiffness tensor is also symmetric in the following sense: $C_{p s}^{i j k m}=C_{p s}^{j k k m}=C_{p s}^{i j m k}=C_{p s}^{k m i j}$. With the constitutive behaviour defined on the physical space, it is now possible through the scaling identities to determine the required law for the tessellated space. This involves the substitution of the relationships $\sigma_{p s}^{i j}=C_{p s}^{i j k m} \varepsilon_{p s k m}$ and $\alpha^{\rho} \partial u_{i p s} / \partial x_{p s}^{j}=\alpha^{u} \beta^{-1} F^{m}{ }_{j} F^{k}{ }_{i} \partial u_{k t s} / \partial x_{t s}^{m}$ into $\alpha^{v} J \sigma_{t s}^{i j}=F_{m}^{i} \sigma_{p s}^{m \ell} F_{\ell}^{j}$ to give

$$
\begin{aligned}
& \sigma_{t s}^{p q}=\left(\alpha^{v} J\right)^{-1} F^{p}{ }_{i} \sigma_{p s}^{i j} F_{j}^{q}=\left(\alpha^{v} J\right)^{-1} F^{p}{ }_{i}\left(C_{p s}^{i j k m} \varepsilon_{p s k m}\right) F_{j}^{q}=\left(\alpha^{v} J\right)^{-1} F^{p}{ }_{i}\left(C_{p s}^{i j k m} \frac{\partial u_{k p s}}{\partial x_{p s}^{m}}\right) F^{q}{ }_{j} \\
& =\left(\alpha^{v} J\right)^{-1} F^{p}{ }_{i}\left(C_{p s}^{i j k m}\left(\alpha^{\rho} \beta\right)^{-1} \alpha^{u} F_{m^{\ell}{ }^{\prime}}{ }_{k} \frac{\partial u_{\ell}}{\partial x_{t s}^{n}}\right) \frac{\partial_{u_{\ell}}}{\partial x_{t s}^{n}} \Gamma^{\cdot}{ }_{j}
\end{aligned}
$$

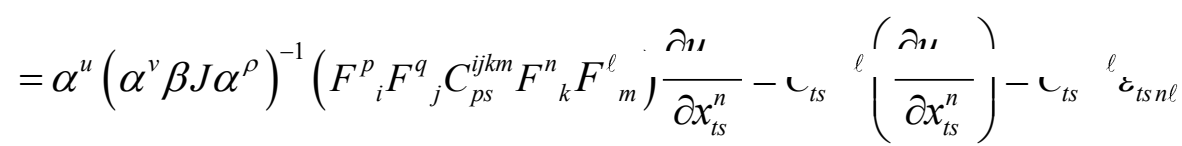

where it can be deduced that $C_{t s}^{p q n \ell}$ possesses the same symmetric properties associated with $C_{p s}^{i j k m}$ alluded to above.

\section{Moment equilibrium}

The conservation of angular momentum gives rise to moment equilibrium, where Equation (4) in the trial space takes the form

$$
-\int_{\Gamma_{t s}^{*}} \underline{r}_{t s} \wedge \underline{\underline{\sigma}}_{t s} \cdot \underline{n}_{t s} d \Gamma_{t s}^{*}+\int_{\Omega_{t s}^{*}} \rho_{t s} \underline{r}_{t s} \wedge \underline{b}_{t s} d V_{t s}^{*}=0
$$

where $\underline{r}_{t s}$ is a position vector and the wedge " $\wedge$ " indicates a cross product. 
Equation (19) does not give rise to an additional independent scalar although the procedure for matching is somewhat involved and different from that presented in Section 4. To illustrate this consider first the application of $p \wedge$ to Equation (19), where $p$ is an arbitrary nonzero constant vector along with substitution of Nanson's identities to reveal

$$
-\int_{\Gamma_{p s}^{*}} \underline{p} \wedge\left(\underline{r}_{t s} \wedge \underline{\underline{\sigma}}_{t s} F^{-T} \cdot \underline{n}_{p s}\right) J d \Gamma_{p s}^{*}+\int_{\Omega_{p s}^{*}} \rho_{t s} \underline{p} \wedge\left(\underline{r}_{t s} \wedge \underline{b}_{t s}\right) J d V_{p s}^{*}=0
$$

Note that the identity $\boldsymbol{d} \boldsymbol{x}_{t s}=F \cdot \boldsymbol{d} \boldsymbol{x}_{p s}$ implies $\underline{r}_{t s}=F \cdot \underline{r}_{p s}$ and recall the identities $\rho_{p s} F \cdot \underline{b}_{p s}=\alpha^{v} \rho_{t s} J \underline{b}_{t s}$ and $\alpha^{v} J \underline{\underline{\sigma}}_{t s} \cdot F^{-T}=F \cdot \underline{\underline{\sigma}}_{p s}$. Moreover, the well-known vector identity $\underline{a} \wedge(\underline{b} \wedge \underline{c})=(\underline{a} \cdot \underline{c}) \underline{b}-(\underline{a} \cdot \underline{b}) \underline{c}$ gives rise to the relationship

$$
\begin{aligned}
\left(F^{-T} \underline{a}\right) \wedge(F \underline{b} \wedge F \underline{c})=\left(F^{-T} \underline{a} \cdot F \underline{c}\right) F \underline{b}-\left(F^{-T} \underline{a} \cdot F \underline{b}\right) F \underline{c}= \\
F[(\underline{a} \cdot \underline{c}) \underline{b}-(\underline{a} \cdot \underline{b}) \underline{c}]=F[\underline{a} \wedge(\underline{b} \wedge \underline{c})]
\end{aligned}
$$

which is needed to relate Equation (20) to its physical-space counterpart.

Consider then setting $\underline{p}=\alpha^{v} F^{-T} \underline{w}$, where by definition $\underline{w}$ is an arbitrary nonzero constant vector and substitution of the various identities into Equation (20), which reveals

$$
\begin{array}{r}
-\int_{\Gamma_{p s}^{*}}\left(F^{-T} \underline{w}\right) \wedge\left(F \underline{r}_{p s} \wedge F \underline{\underline{\sigma}}_{p s} \cdot \underline{n}_{p s}\right) d \Gamma_{p s}^{*}+\int_{\Omega_{p s}^{*}} \rho_{p s}\left(F^{-T} \underline{w}\right) \wedge\left(F \underline{r}_{p s} \wedge F \underline{b}_{p s}\right) d V_{p s}^{*}= \\
-F \int_{\Gamma_{p s}^{*}} \underline{w} \wedge\left(\underline{r}_{p s} \wedge \underline{\underline{\sigma}}_{p s} \cdot \underline{n}_{p s}\right) d \Gamma_{p s}^{*}+F \int_{\Omega_{p s}^{*}} \rho_{p s} \underline{w} \wedge\left(\underline{r}_{p s} \wedge \underline{b}_{p s}\right) d V_{p s}^{*}=0
\end{array}
$$

which is a weak relationship for moment equilibrium in the two spaces.

In summary the analysis above provides the relationship

$$
\alpha^{v}\left(F^{-T} \underline{w}\right) \wedge\left[-\int_{\Gamma_{t s}^{*}} \underline{r}_{t s} \wedge \underline{\underline{\sigma}}_{t s} F^{-T} \cdot \underline{n}_{t s} d \Gamma_{t s}^{*}+\int_{\Omega_{t s}^{*}} \rho_{t s} \underline{r}_{t s} \wedge \underline{b}_{t s} d V_{t s}^{*}\right]=
$$




$$
-F\left[\underline{w} \wedge\left(\int_{\Gamma_{p s}^{*}}\left(\underline{r}_{p s} \wedge \underline{\underline{\sigma}}_{p s} \cdot \underline{n}_{p s}\right) d \Gamma_{p s}^{*}+\int_{\Omega_{p s}^{*}} \rho_{p s}\left(\underline{r}_{p s} \wedge \underline{b}_{p s}\right) d V_{p s}^{*}\right)\right]=0
$$

where $\underline{w}$ is an arbitrary nonzero constant vector.

In addition and following on from the movement definition of Equation (11), is the first moment-of-movement, which is defined here in the two spaces to be

$$
\underline{M}_{p s}^{1}=\int_{\Omega_{p s}^{*}} \rho_{p s} \underline{r}_{p s} \wedge \underline{u}_{p s} d V_{p s}^{*} \text { and } \underline{M}_{t s}^{1}=\int_{\Omega_{t s}^{*}} \rho_{t s} \underline{r}_{t s} \wedge \underline{u}_{t s} d V_{t s}^{*}
$$

where the superscript "1" indicates first moment.

Substitution of Nanson's identity $d V_{t s}=J d V_{p s}, \underline{r}_{t s}=F \cdot \underline{r}_{p s}$ and $\rho_{p s} \underline{u}_{p s}=\alpha^{u} \rho_{t s} J F^{-1} \cdot \underline{u}_{t s}$ along with the application of $\alpha^{u} F^{-T} \underline{w} \wedge$ to Equation (24) gives

$$
\begin{aligned}
\alpha^{u}\left(F^{-T} \underline{w}\right) \wedge \underline{M}_{t s}^{1}=\int_{\Omega_{p s}^{*}} \rho_{p s}\left(F^{-T} \underline{w}\right) \wedge\left(F \underline{r}_{p s} \wedge F \underline{u}_{p s}\right) d V_{p s}^{*}= \\
\quad F \int_{\Omega_{p s}^{*}} \rho_{p s} \underline{w} \wedge\left(\underline{r}_{p s} \wedge \underline{u}_{p s}\right) d V_{p s}^{*}=F\left[\underline{w} \wedge \underline{M}_{p s}^{1}\right]
\end{aligned}
$$

where the arbitrariness of $\underline{w}$ enforces the weak identity and defines the sense in which $\underline{M}_{p s}^{1}$ and $\underline{M}_{t s}^{1}$ are related; similar relationships follow for higher-order moments.

\section{Procedural considerations}

This section is concerned with the procedure for initiating, testing and verifying the Tessellated Continuum Mechanics (TCM) approach. This is achieved through direct application of mechanics principles to physical structures represented by pre-fractals and on contrasting with results from the tessellated approach. Verification is first performed on a 1-D structure represented by pre-fractals for the Cantor Set. Testing a 1-D structure is 
beneficial as its simplicity enables the theory to be readily understood and appreciated, which would not be the case for more complex structures. Bending is considered to demonstrate that fully continuum structures can indeed represent the behaviour of discontinuous although admittedly in this case for a 1-D structure, of some simplicity. In 2D, two plates are investigated represented by pre-fractals for the classical Sierpinski Carpet and the possibly less familiar Vicsek fractal. The Sierpinski Carpet provides the type of perforated plate-like structure that the work is purposely designed to accommodate. The inclusion of the Vicsek fractal is solely to demonstrate the capability of the theory in providing exact solutions to plate structures that are some distance from a continuous rectangular plate.

\subsection{Procedure for application of the tessellated approach}

Presented in brief in this section are the procedural steps for implementation of the tessellated approach.

\subsubsection{Step 1-Tessellation formation}

Recall from Section 2 that although pre-fractals and tessellations are created independently using disparate iterated function schemes (IFS), where the contraction maps involved in the tessellations are constructed to close holes in $\hat{E}_{1}=\bigcup_{i} S_{i}\left(\hat{E}_{0}\right)$. This involves placing an initial triangular tessellation on $\hat{E}_{0}=\hat{T}_{0}$ followed by forming $\hat{E}_{1}$, which includes a scaled replica of the initial tessellation on each pre-fractal element of $\hat{E}_{1}$, i.e. on $S_{i}\left(\hat{E}_{0}\right)$. The initial tessellation on $\hat{E}_{0}=\hat{T}_{0}$, although not unique, can also be used as a means of mesh refinement in any finite element analysis but sufficient elements are required to enable holes to be closed on $\hat{E}_{1}$ to form $\hat{T}_{1}$. The procedure is illustrated in 
Figure 2 and can be imagined to involve the morphing of $\hat{E}_{1}$ into $\hat{T}_{1}$ by means of tile distortion, where selected triangular tiles are stretched linearly in order to close the holes in $\hat{E}_{1}$. Once $\hat{T}_{1}$ is established and recognising that the number of tiles on $\hat{E}_{1}$ and $\hat{T}_{1}$ are identical it is then possible to form the maps $P_{i}$ to satisfy the relationship $\hat{T}_{1}=\bigcup_{i} P_{i}\left(\hat{T}_{0}\right)$. Note that each contraction map $P_{i}$ is formed by a collection of linear sub-maps each designed to map a tile in $\hat{T}_{0}$ to a corresponding tile in $\hat{T}_{1}$. Once the maps $P_{i}$ are known it is then a matter of simple recursion to create $\hat{T}_{k}$ as described in Section 2.

\subsubsection{Step 2-Direct mapping}

Once $\hat{T}_{k}$ and $\hat{E}_{k}$ are known and appreciating that each has the exact same number of linear tiles it becomes possible to form the hole-file map. As mentioned in Section 2 for numerical work a direct procedure of identifying corresponding elements in $\hat{T}_{k}$ and $\hat{E}_{k}$ is preferred. For any two corresponding elements, a linear map taking points between them can be readily obtained. Under the finite similitude theory, a map taking points in an element in $\hat{E}_{k}$ to a corresponding element in $\hat{T}_{k}$ is viewed as space distortion. Invariably elements in $\hat{T}_{k}$ are bigger or equal to corresponding elements in $\hat{E}_{k}$, so the imagined distortion process is one of local piecewise expansion. A spatial increment in the physical space (where the pre-fractal resides) is related to an increment in the tessellated space (where the tessellation resides) by the relationship $\boldsymbol{d} \boldsymbol{x}_{t s}=F \cdot \boldsymbol{d} \boldsymbol{x}_{p s}$, where $F$ is the hole-fill map and plays a pivotal role in the similitude theory as discussed in Section 5.

It is important to appreciate that in practical terms the use of a piecewise-linear hole-fill map limits perforations to planar surfaces. This is required so that holes can be collapsed without gaps appearing in the final tessellation. Thus a plate consisting of circular 
perforations for example would typically require the circles to be approximated first by means of regular polygons.

\subsubsection{Step 3-Material properties}

The theory outlined in Section 5 incorporates a number of scaling parameters that must be set prior to application of the method, i.e. $\alpha^{\rho}, \alpha^{u}, \alpha^{v}$ and $\alpha^{e}$ although $\alpha^{e}=\left(\alpha^{\rho} \beta\right)^{-1} \alpha^{v} \alpha^{u}$, so $\alpha^{e}$ is not an independent parameter. The tessellated approach has a peculiar advantage in that analysis results for the physical space (where the pre-fractal resides) is invariably of principal interest in any investigation. In this setting any results obtained on a tessellation serve only as a vehicle to obtain results on a corresponding prefractal. This means that the scaling parameters $\alpha^{\rho}, \alpha^{u}$ and $\alpha^{v}$ can be set to any arbitrary value without affecting the results in the physical space. This can be contrasted against the typical setting for the finite-similitude theory applied to scaled experimentation, where in this case results in the physical and scaled experiment have significance and constrain the parameters involved. Although the parameters $\alpha^{\rho}, \alpha^{u}$ and $\alpha^{v}$ can be arbitrary it is of interest to consider here those that imbue the tessellated space with physical attributes. Equation (9) for example provides a connection between the densities in each space, so setting $\alpha^{\rho}=J^{-1}$ in the relationship $\rho_{p s}=\alpha^{\rho} J \rho_{t s}$ provides $\rho_{p s}=\rho_{t s}$, where $J=\operatorname{det} F$. Note here that the hole-fill map $F$ varies spatially in a piecewise-constant manner and consequentially in general $\alpha^{\rho}$ takes up different values on each tile in a tessellation. A slightly more common alternative when dealing with fractals is mass conservation and since Equation (8) provides $M_{p s}=\alpha^{\rho} M_{t s}$, mass is matched with $\alpha^{\rho}=1$, which in this case applies to the whole tessellation. These choices for $\alpha^{\rho}$ have a consequential impact on the choices for $\alpha^{u}$ and $\alpha^{v}$, if similitude is to be enforced. Equation (12) in the form $\alpha^{\rho} \underline{u}_{p s}=\alpha^{u} F^{-1} \cdot \underline{u}_{t s}$ reduces to the finite similitude condition $\underline{u}_{p s}=F^{-1} \cdot \underline{u}_{t s}$ on setting $\alpha^{u}=\alpha^{\rho}$ 
, which means $\alpha^{u}$ takes up the values $J^{-1}$ or 1 in line with $\alpha^{\rho}$. The parameter $\alpha^{v}$ appears in Equation (10) and indirectly in Equation (14) but with $\alpha^{u}=\alpha^{\rho}$ it follows also that $\alpha^{e}=\left(\alpha^{\rho} \beta\right)^{-1} \alpha^{v} \alpha^{u}=\alpha^{v} \beta^{-1}$. Therefore energy density matching in Equation (14) is achieved with $\alpha^{v} \beta^{-1}=1$, i.e. $\alpha^{v}=\beta$.

A feature of finite similitude is the matching of important material properties and Young's modulus and Poisson ratio play an important role in tessellated-plate deformation through the relationship

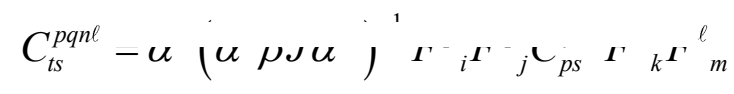

arising from Equation (18).

Note here that $C_{p s}^{i j k m}$ is fixed in Equation (26) by the material of the physical pre-fractal plate but $C_{t s}^{p q n \ell}$ is influenced by the hole-fill map $F$ along with the product $\alpha^{u}\left(\alpha^{v} \alpha^{\rho}\right)^{-1}$. Thus, the tessellation can be considered to be formed by tiles of different materials depending on this product and $F$. A tessellation of a single material tends only to be practically possible in $1-\mathrm{D}$, where the hole-fill map is sufficiently simple to ensure that the identity $\alpha^{u}\left(\alpha^{v} \alpha^{\rho}\right)^{-1} F$ is uniform.

\subsubsection{Step 4-Boundary conditions}

In order to perform an analysis of the tessellated plate, it is necessary to apply appropriate boundary conditions. The boundary conditions are those on the pre-fractal but transferred to the tessellation under the scaling identities. Displacement is transferred under the relationship $\alpha^{\rho} \underline{u}_{p s}=\alpha^{u} F^{-1} \cdot \underline{u}_{t s}\left(\right.$ or $\left.\alpha^{\rho} \underline{u}_{p s}=\alpha^{u} \beta^{-1} F^{T} \cdot \underline{u}_{t s}\right)$, where for example a fixedboundary displacement constraint on a pre-fractal immediately transfers as a fixed displacement constraint on the tessellation. Similarly for stress constraints, Equation (12) 
applies (i.e. $\underline{\underline{\sigma}}_{p s}=\alpha^{v} J F^{-1} \cdot \underline{\underline{\sigma}}_{t s} \cdot F^{-T}$ ), which confirms that free-stress boundary conditions match in each space. This is particularly pertinent for perforations, which are stress-free in the physical space and must, therefore, remain stress-free in the tessellated space despite no gaps appearing at element edges in a tessellation. Moment boundary conditions are applicable to beams and plates, but care is required to ensure that moments are related in accordance with the relationships outlined in Section 6.

\subsubsection{Step 5-Analysis and post-processing}

Following creation of a tessellated structure and following application of boundary conditions (which can involve free stress on some internal element edges) it is a simple matter to run a traditional Galerkin finite element analysis. All fields on a tessellation can subsequently be lifted back to the pre-fractal using the scaling identities.

\section{Case Studies in 1-D}

It is of interest to examine the application of the tessellated theory in a 1-D setting as this reduces the relative complexity of the theory and allows both numerical and analytical investigations to be performed. The pre-fractals considered are those applied in the construction of the classical Cantor-dust fractal. One of the difficulties with holey 1-D constructions is that they do not form load-bearing structures and consequentially cannot withstand load. To rectify this rigid elements are assumed to occupy the holes in the prefractals. Although somewhat artificial this assumption is particularly convenient for the tessellation as it immediately means slopes match for elements adjoining the discontinuity network (see Figure 1).

The 1-D models are built according to the procedure outlined in Section 2. The material properties along with geometrical features of the pre-fractal and tessellated models used in this study are depicted in Table 1. Both the pre-fractal and tessellated geometry are created 
by the application of contraction maps given in Section 2.1. Three levels of complexity are tested with analysis for the pre-fractals $\hat{E}_{1}, \hat{E}_{2}$ and $\hat{E}_{3}$ performed on the tessellations $\hat{T}_{1}, \hat{T}_{2}$ and $\hat{T}_{3}$ identified in Table 1 under the headings $k=1,2$ and 3. In addition, isotropic and anisotropic scaling is tested, distinguished by their hole-fill maps, which are:

$F_{k}=\beta_{k}\left[\begin{array}{ccc}1 & 0 & 0 \\ 0 & 1 & 0 \\ 0 & 0 & 1\end{array}\right], F_{k}=\left[\begin{array}{ccc}\beta_{k} & 0 & 0 \\ 0 & 1 & 0 \\ 0 & 0 & \beta_{k}\end{array}\right]$ and $F_{k}=\left[\begin{array}{ccc}\beta_{k} & 0 & 0 \\ 0 & \sqrt{\beta_{k}} & 0 \\ 0 & 0 & \beta_{k}^{2}\end{array}\right]$

where $\beta_{k}=(3 / 2)^{k}$ and $F_{k}$ is the hole-fill map connecting $\hat{E}_{k}$ to $\hat{T}_{k}$.

The hole-fill maps in Equation (27) are uniform and when applied to $\hat{E}_{k}$ have the effect of closing the holes whilst at same time maintaining the overall initial length of the structure. The material properties for the tessellation are determined using Equations (9) and (26), which are presented in Table 1 along with associated properties for the pre-fractal. The scaling factors $\alpha^{\rho}, \alpha^{u}$ and $\alpha^{v}$, which affect the predictions on the tessellations, are listed in Tables 1 and 2.

All numerical analysis performed is done with commercial FE software ABAQUS (version 6.13), using linear-beam elements, type B32 [31] for both pre-fractal and tessellated structures.

\subsection{Analytical verification}

In order to test the efficacy of the ABAQUS numerical model, comparison is made against an analytically derived solution for $\hat{E}_{k}$ isotropic and anisotropic scaling with $\beta_{k}=(3 / 2)^{k}$. A Bernoulli cantilever beam (i.e. a beam with clamped-free (CF) boundary conditions) is considered with a lateral load of magnitude $Q_{p s}$ applied to the free end. The equivalent tessellated model is correspondingly a CF beam, but in this case, the covariant displacement is required to satisfy the relationship $\alpha^{\rho} \underline{u}_{p s}=\alpha^{u} \beta^{-1} F^{T} \cdot \underline{u}_{p s}$, which for 
$\alpha^{\rho}=\alpha^{u}=1$ and with $F=F_{k}$ taking any of the forms in Equation (27) gives $w_{t s}=\beta F_{33}^{-1} w_{p s}$ for lateral displacement in the z-direction, where $F_{33}=\beta_{k}$ or $F_{33}=\beta_{k}^{2}$. Both beams are presented in Figure 3 on $\hat{E}_{1}$ for the pre-fractal and on $\hat{T}_{1}$ for the tessellation.

Each Cantor set structure $\hat{E}_{k}$ consists of $2^{k}$ deformable parts of equal length and for $k>1$ includes $k(k-1)+1$ rigid segments (holes) as depicted in Figure 1(a). Enumerating nodes from left to right (starting from $i=1$ ) results in a segment placed between coordinates $x_{p s}^{i}$ and $x_{p s}^{i+1}$ being rigid for even $i$ and deformable if $i$ is odd. The Bernoulli cantilever beam requires a solution to $w_{p s}^{\prime \prime}=M_{x_{p s}} / E_{p s} I_{p s}$, where $M_{x_{p s}}=Q_{p s}\left(\ell \ldots, E_{p s}\right.$ is Young's modulus, $I_{p s}$ is the second moment of area and $\ell$ is the beam length. Thus deformation in any deformable segment is a cubic polynomial and takes the general form

$\left.w_{p s}\left(\hat{x}_{p s}\right)=\frac{Q_{p s} \ell^{-}}{E_{p s} I_{p s}} \mid \frac{\left.-\hat{x}_{p s}^{i}\right)^{2}}{2}-\frac{\left(\hat{x}_{p s}-\hat{x}_{p s}^{i}\right)^{3}}{6}\right)+\left.\frac{d w_{p s}}{d \hat{x}_{p s}}\right|_{x_{p s}^{i}}\left(\hat{x}_{p s}-\hat{x}_{p s}^{i}\right)+\left.w_{p s}\right|_{x_{p s}^{i}}$

where $\hat{x}_{p s}=x_{p s} / \ell$ and $x_{p s}^{i} \leq x_{p s} \leq x_{p s}^{i+1}$.

A rigid element satisfies the equation $w_{p s}^{\prime \prime}=0$ and takes the form

$w_{p s}\left(\hat{x}_{p s}\right)=\left.\frac{d w_{p s}}{d \hat{x}_{p s}}\right|_{x_{p s}^{i}}\left(\hat{x}_{p s}-\hat{x}_{p s}^{i}\right)+\left.w_{p s}\right|_{x_{p s}^{i}}$

which is readily obtained from Equation (28) in the limit $E_{p s} \rightarrow \infty$.

These solutions for displacement and slope for different pre-fractals are presented in Figure 4. Included in the figure are ABAQUS predictions, which provide almost exact replication of the analytical output. An alternative route to analytical solutions on $\hat{E}_{k}$ is via the similitude theory and the lifting of these from $\hat{T}_{k}$. This can be achieved by first 
determining the solution to $w_{t s}^{\prime \prime}=M_{x_{s s}} / E_{t s} I_{t s}$, where $M_{x_{t s}}=Q_{t s}\left(\ell,, E_{t s}\right.$ is the Young's modulus; $I_{t s}$ is the second moment of area and $\ell$. is the beam length. The tessellated beam is absent of rigid segments (as these appear as discontinuities) and the deformable segments are described by an equation similar in form to (28), i.e.

$\left.w_{t s}\left(\hat{x}_{t s}\right)=\frac{Q_{t s} \ell^{-}}{E_{t s} I_{t s}} \frac{\left.\hat{x}_{t s}^{i}\right)^{2}}{2}-\frac{\left(\hat{x}_{t s}-\hat{x}_{t s}^{i}\right)^{3}}{6}\right)+\left.\frac{d w_{t s}}{d \hat{x}_{t s}}\right|_{x_{t s}^{i}}\left(\hat{x}_{t s}-\hat{x}_{t s}^{i}\right)+\left.w_{t s}\right|_{x_{t s}^{i}}$

where $\hat{x}_{t s}=x_{t s} / \ell$ and $x_{t s}^{i} \leq x_{t s} \leq x_{t s}^{i+1}$.

The lifting of Equation (30) to arrive at Equation (28) is achieved on substitution of the following maps and identities: $Q_{t s}=\left(\alpha^{v}\right)^{-1} F_{33} Q_{p s}=\beta^{-1} F_{33} Q_{p s}, \quad \ell_{0} \mapsto F_{11} \ell_{0}, \quad I_{t s}=F_{22} F_{33}^{3} I_{p s}$, $\hat{x}_{t s}-\hat{x}_{t s}^{i} \mapsto \hat{x}_{p s}-\hat{x}_{p s}^{i}, w_{t s}=\beta F_{33}^{-1} w_{p s}$ and from Equation (26) $E_{t s}=\beta^{-2} J^{-1} F_{11}^{4} E_{p s}=\beta^{-2} F_{11}^{3} F_{22}^{-1} F_{33}^{-1} E_{p s}$, since $J=\beta^{3}=F_{11} F_{22} F_{33}$.

An alternative approach to confirm Equation (28) and (30) are related is directly from the governing equation $w_{t s}^{\prime \prime}=M_{x_{s s}} / E_{t s} I_{t s}$ and $w_{p s}^{\prime \prime}=M_{x_{p s}} / E_{p s} I_{p s}$, which on substitution of pertinent identities takes the form

$\frac{d^{2} w_{t s}}{d x_{t s}^{2}}=\frac{\beta}{F_{11}^{2} F_{33}} \frac{d^{2} w_{p s}}{d x_{p s}^{2}}=\frac{M_{x_{t s}}}{E_{t s} I_{t s}}=\frac{\beta^{-1} F_{11} F_{33} M_{x_{p s}}}{\left(\beta^{-2} F_{11}^{3} F_{22}^{-1} F_{33}^{-1} E_{p s}\right)\left(F_{22} F_{33}^{3} I_{p s}\right)}=\frac{\beta}{F_{11}^{2} F_{33}} \frac{M_{x_{p s}}}{E_{p s} I_{p s}}$

where use is made of the identity $M_{x_{s s}}=\beta^{-1} F_{11} F_{33} M_{x_{p s}}$, which confirms that with similitude the solution to $w_{t s}^{\prime \prime}=M_{x_{t s}} / E_{t s} I_{t s}$ is also a solution to $w_{p s}^{\prime \prime}=M_{x_{p s}} / E_{p s} I_{p s}$ and vice versa.

ABAQUS results for the tessellation for different values of $k$ are depicted in Figure 5. It is clear from the Figures 4 and 5 that very precise predictions are achievable with ABAQUS on both pre-fractals and tessellations. Now that the tessellated approach has been confirmed analytically for a simple cantilever beam and the accuracy of the ABAQUS 
models confirmed it remains to test the lifting process for both isotropic and anisotropic cases in Equation (27).

\subsection{Isotropic lifting process}

The beam depicted in Figure 3 is reanalysed numerically for isotropic scaling to test the lifting of results and the Python code that has been specifically written to enable ABAQUS to be interfaced with pre-fractal structures. The results on a pre-fractal can be obtained by two independent routes, i.e. by means of direct analysis and indirectly by lifting results from the tessellation. The lifting process is of principal interest here to illustrate how it is possible to achieve results of high accuracy on a continuum. However, the fact that two routes of analysis are possible provides a direct means for checking accuracy even when analytical solutions are unavailable although in this particular instance these are presented in Figure 6. The lifted and direct numerical predictions are provided in Figure 6 on $\hat{E}_{k}$ for $k=1: 3$. Note from Figure 6 that the maximum absolute errors involved are extremely small but it is important to appreciate that numerical predictions are being contrasted against numerical predictions. Thus, some care is required here because with similitude, and with the same number of elements employed in both analyses, extremely small errors are anticipated yet absolute errors can (with a coarse mesh) remain large. The results of Figures 4 and 6 confirm that absolute errors are small in this case also. The tests confirm the equivalence of the direct and the tessellated approach.

\subsection{Anisotropic lifting process}

This section is concerned with anisotropic scaling of pre-fractal elements for the formation of a tessellation for the determination of lateral beam deformation of the $\mathrm{CC}$ beam depicted in Figure 7. The beam is loaded by an off-centre point load, located at the left edge of the central rigid segment (hole). Note that the central rigid segment (hole) is 
present for all $\hat{E}_{k}$ for all $k \geq 1$, so the load-point position does not change with $k$. The two anisotropic hole-fill maps presented in Equation (27) are applied. The tests are designed to illustrate that anisotropic scaling is permissible and that predicted results on the pre-fractals (lifted from a tessellation) do not depend on the form of the hole-fill map. The material properties of the pre-fractal are identical to those used in Sections 8.1 and 8.2 and details are provided in Table 2. Note that Young's modulus is for convenience set to unity for the pre-fractal material since dimensionless outputs are of principal interest. The density and elastic properties for the tessellated material are determined using Equations (9) and (26), respectively and are recorded in Table 2 . The predictions for the tessellated beams on $\hat{T}_{3}$ are presented in Figure 8. It is apparent on examination of the figure that both (anisotropically-scaled) beams provide near identical outputs despite having different widths and heights. Recall that each tessellated beam is created on application of the anisotropic scaling maps in Equation (27) to the pre-fractal elements, which has the effect of producing different lateral dimensions. The reason for the near identical results in Figure 8 is not because displacements are identical but as a consequence of the dimensionless ordinates used on the axes of this figure. The displacement and slope of the pre-fractal CC beam on $\hat{E}_{3}$ can be found in Figure 9. Observe that three methods of prediction are presented, i.e. the direct method, and the two lifted tessellated results obtained on employing the two anisotropic maps in Equation (27). All methods provide consistent results to very high accuracy and the source of error can be associated with rounding errors in the numerical calculations. For consistency the number of finite elements used in each deformable pre-fractal segment (and tile) was set equal to 11 as this was found to provide good accuracy. This number or elements was applied for the entire numerical analysis of the beam structures. 


\section{Case Studies in 2-D}

The main focus of this work is the analysis of 2-D pre-fractal plate structures since these are found in engineering applications as discussed in the introduction. The pre-fractals analysed here are employed in the construction of two well-known traditional fractals, i.e. the Sierpinski Carpet and Vicsek fractal. Unlike the 1-D models considered in Section 8 there is no need for the employment of artificial rigid elements since holey plates are loadbearing structures. Three different levels of complexity are analysed with the meshes employed in the analysis of the Sierpinski carpet found in Figure 2 for both pre-fractals and tessellations. It is important to appreciate that the meshes depicted in this figure are generated as part of the recursive procedure for the generation of pre-fractals and tessellations. In this case an initial tessellation consisting of eight elements was placed on $\hat{E}_{0}$ and $\hat{T}_{0}$. The number of elements on $\hat{E}_{1}$ and $\hat{T}_{1}$ is correspondingly equal to the number of elements on $\hat{E}_{0}$ multiplied by the number of contraction maps used in the IFS, which for the Sierpinski carpet and Vicsek is eight and five, respectively. The number of elements employed in the analysis of both the Sierpinski and Vicsek pre-fractals is given in Table 3. The contraction maps employed are listed in Appendices A and B (see Tables A1 and B1) for the Sierpinski Carpet and Vicsek fractal, respectively. Material properties and dimensions are presented in Table 4, with both the Sierpinski and Vicsek sharing the same material properties and overall dimensions. Likewise the contraction maps used to create corresponding tessellated models for both structures are provided in Appendices A and B (see Tables A2 and B2), respectively. Note that both pre-fractals and tessellations involve the same number of contraction maps.

The hole-fill maps for the 2-D plates generally involve off-diagonal terms to account for shear and take the form 
$F_{k}=\left[\begin{array}{ccc}F_{11} & F_{12} & 0 \\ F_{21} & F_{22} & 0 \\ 0 & 0 & F_{33}\end{array}\right]_{k}$

where unlike the 1-D cases considered in Section $8, F_{k}$ is seldom uniform as hole-filling is an inhomogenous process.

The determination of the hole-fill maps is achieved using the object-orientable programing language Python, which facilitates the transfer of data to the ABAQUS software. Unlike the 1-D analysis, analytical solutions are unavailable, so the tessellated lifted solutions are contrasted against direct numerical analysis performed in the commercial FE software ABAQUS. Shell-elements, type SR8 [31], are used to model the structures in both the tessellated and pre-fractal spaces.

\subsection{Numerical Verification of Sierpinski Carpet}

The Sierpinski carpet pre-fractals presented in Figure are considered as holey-plates with CCCC boundary conditions subject to lateral pressure of magnitude $R_{p s}$. The corresponding tessellated structure possesses identical boundary conditions but a pressure of magnitude $R_{t s}$, which varies over the surface of the tessellation determined by Equation (10) with $F$ substituted by $F_{k}$ of Equation (32). Although the density of the plate plays no significant part in the static analysis it is nonetheless determined using Equation (9). Equation (26) plays a critical role in determining the elastic properties of the tiles in the tessellation. The distribution of the normal $E_{11}$ values of Young's moduli on the tessellation (as determined by Equation (26)) is presented in Figure 10. The figure reveals just how geometric complexity from a pre-fractal is transferred into material complexity on a tessellation. Although a uniform pressure is applied on the pre-fractal plate the pressure over the tessellated palate as calculated using Equation (10) takes on a more complex pattern as 
apparent on examination Figure 11. Confirmation of the validity of the approach is achieved on comparison of the results between the pre-fractal responses in terms of lateral deformation contrasted to the lifted response from the tessellated plate. These results are presented in Figures 12 and 13, where the lifted results are almost identical to the direct pre-fractal predictions within the bounds of rounding errors in the numerical calculations. An overall picture of the lateral behaviour of the tessellated and pre-fractal plates is presented in Figure 14.

\subsection{Processing time analysis on Sierpinski Carpet}

Although the prime focus of the work is to determine whether it is possible to analysis perforated-plates to high accuracy it is interest to investigate the extent of processing and computing time discrepancies between the two approaches. The processing times required to build the pre-fractals and corresponding tessellations are presented in Figure 15. Not too unexpectedly processing time for model construction for the tessellated structures is slightly longer than the direct approach due to the time required determining the element stiffness matrices. Note however that corresponding pre-fractals and tessellations have identical numbers of unknowns and equations.

\subsection{Numerical Verification of Vicsek fractal}

The Vicsek pre-fractals presented in Figure 16 are selected to provide a significant challenge for the tessellated approach. These pre-fractals are significantly different in form from a continuous-rectangular plate and at first glance it might be considered impractical if not impossible to analyse these types of structure on a continuous plate. However, the tessellated approach is able to achieve this to very high accuracy. The meshes used in the analysis are depicted in Figure 16, which are generated by the recursive process with again an initial tessellation of 8 tiles on $\hat{E}_{0}$ and $\hat{T}_{0}$. Note the severity of the skewed elements that 
appear on the tessellations. Numerical issues typically associated with skewed elements do not arise with the tessellated approach because skewness and deviation in material properties counteract each other to represent the behaviour on the pre-fractals. Both the pre-fractals and tessellation are under CCCC boundary conditions although only the four outermost edges of the pre-fractals are clamped. A displacement boundary condition is applied on the centre of each model. Contour plots for the pre-fractal structure $\hat{E}_{3}$ with its corresponding tessellation $\hat{T}_{3}$, are represented in Figure 17 for lateral deformation and slope. Particular differences are highlighted along the midline of the Vicsek in Figures 18 and 19 in terms of out-of-plane deformation and slope. It is evident from these figures that high accuracy is achievable with the tessellated approach.

\section{Conclusions}

In this paper validation the tessellated method is achieved by means of testing the static response of holey pre-fractal structures of different levels of complexity. This is achieved by ensuring that the behaviour of pre-fractal elements and tiles correspond. The study has led to the following conclusions:

- A transport approach for static analysis of fractals in 1-D and 2-D has been established.

- The tessellated theories linking pre-fractal structures and tessellations for static analysis have been established and verified.

- The presented approach has been verified analytically and numerically to confirm that perforated structures can be analysed to high accuracy on continua.

- Different pre-fractal structures have been investigated and the validity and accuracy of the tessellated approach has been confirmed for these structures.

- The tessellated approach has been shown to be able to predict static responses of beams and plates under different boundary and loading conditions. 


\section{Appendix A: Maps for the Sierpinski carpet}

The mapping results on the pre-fractal and the tessellated space should be used the mapping listed in Table A1 and Table A2. The coordinates used in the tables are dimensionless and for a square plate take the form $x=x_{p s} / \ell$ and $y=y_{p s} / \ell$. (or $x=x_{t s} / \ell$. and $y=y_{t s} / \ell$. . The same number of maps ( 8 in this case) is used in the creation of the Sierpinski carpet and its associated tessellation. Only four tessellated contraction maps are presented in Table A2 with the missing four being identical to corresponding pre-fractals contraction maps, i.e. $\mathrm{P}_{i}=\mathrm{S}_{i}$ for $i=1,3,6,8$.

Table A1. Contraction maps for Sierpinski Carpet [21].

\begin{tabular}{|c|c|}
\hline $\mathrm{S}_{1}(x, y)=\left(\frac{x}{3}, \frac{y}{3}\right)$ & $\mathrm{S}_{2}(x, y)=\left(\frac{1}{3}+\frac{x}{3}, \frac{y}{3}\right)$ \\
\hline $\mathrm{S}_{3}(x, y)=\left(\frac{2}{3}+\frac{x}{3}, \frac{y}{3}\right)$ & $\mathrm{S}_{4}(x, y)=\left(\frac{x}{3}, \frac{1}{3}+\frac{y}{3}\right)$ \\
\hline $\mathrm{S}_{5}(x, y)=\left(\frac{2}{3}+\frac{x}{3}, \frac{1}{3}+\frac{y}{3}\right)$ & $\mathrm{S}_{6}(x, y)=\left(\frac{x}{3}, \frac{2}{3}+\frac{y}{3}\right)$ \\
\hline $\mathrm{S}_{7}(x, y)=\left(\frac{1}{3}+\frac{x}{3}, \frac{2}{3}+\frac{y}{3}\right)$ & $\mathrm{S}_{8}(x, y)=\left(\frac{2}{3}+\frac{x}{3}, \frac{2}{3}+\frac{y}{3}\right)$ \\
\hline
\end{tabular}

Table A2. Contraction maps for the tessellation to mirror the Sierpinski Carpet [21].

\begin{tabular}{|l|ll|}
\hline \multirow{2}{*}{$\mathrm{P}_{2}(x, y)$} & $\mathrm{P}_{21}(x, y)=\left(\frac{1}{3}+\frac{x}{3}, \frac{1}{3}+\frac{y}{3}\right)$ & if $y \geq 2 x$ and $1 / 3 \leq x \leq 1 / 2$ \\
\cline { 2 - 3 } & $\mathrm{P}_{22}(x, y)=\left(\frac{1}{3}+\frac{x}{3}, \frac{1}{3}-\frac{x}{3}+\frac{y}{3}\right)$ & if $y \geq 2(1-x)$ and $1 / 2 \leq x \leq 2 / 3$ \\
\cline { 2 - 3 } & $\mathrm{P}_{23}(x, y)=\left(\frac{x}{2}, \frac{1}{3}+\frac{y}{3}\right)$ & $\begin{array}{l}\text { if } y \geq 2 x \text { and } 1 / 3 \leq x \leq 1 / 2 \text { or } \\
\text { if } y \geq 2(1-x) \text { and } 1 / 2 \leq x \leq 2 / 3\end{array}$ \\
\hline $\mathrm{P}_{4}(x, y)$ & $\mathrm{P}_{41}(x, y)=\left(\frac{1}{3}+\frac{x}{3}, \frac{1}{3}+\frac{y}{3}\right)$ & if $y \leq x / 2$ and $2 / 3 \leq x \leq 1$ \\
\hline
\end{tabular}




\begin{tabular}{|c|c|c|}
\hline & $\mathrm{P}_{42}(x, y)=\left(\frac{1}{3}+\frac{x}{3}-\frac{y}{3}, \frac{1}{3}+\frac{y}{3}\right)$ & if $y \geq(1-x / 2)$ and $2 / 3 \leq x \leq 1$ \\
\hline & $\mathrm{P}_{43}(x, y)=\left(\frac{x}{2}, \frac{1}{3}+\frac{y}{3}\right)$ & $\begin{array}{l}\text { if } y \geq x / 2 \text { and } 2 / 3 \leq x \leq 1 \text { or } \\
\text { if } y \geq(1-x / 2) \text { and } 2 / 3 \leq x \leq 1\end{array}$ \\
\hline \multirow{3}{*}{$\mathrm{P}_{5}(x, y)$} & $\mathrm{P}_{51}(x, y)=\left(\frac{2}{3}+\frac{x}{3}-\frac{y}{3}, \frac{1}{3}+\frac{y}{3}\right)$ & if $2 y \leq 1-x$ and $0 \leq x \leq 1 / 3$ \\
\hline & $\mathrm{P}_{52}(x, y)=\left(\frac{1}{3}+\frac{x}{3}+\frac{y}{3}, \frac{1}{3}+\frac{y}{3}\right)$ & if $2 y \geq 1-x$ and $0 \leq x \leq 1 / 3$ \\
\hline & $\mathrm{P}_{53}(x, y)=\left(\frac{1}{2}+\frac{x}{2}, \frac{1}{3}+\frac{y}{3}\right)$ & $\begin{array}{l}\text { if } 2 y \geq 1-x \text { and } 0 \leq x \leq 1 / 3 \text { or } \\
\text { if } 2 y \leq 1-x \text { and } 0 \leq x \leq 1 / 3\end{array}$ \\
\hline \multirow{3}{*}{$\mathrm{P}_{7}(x, y)$} & $\mathrm{P}_{71}(x, y)=\left(\frac{1}{3}+\frac{x}{3}, \frac{2}{3}-\frac{x}{3}+\frac{y}{3}\right)$ & if $y \leq 1 / 2-x / 2$ and $1 / 3 \leq x \leq 1 / 2$ \\
\hline & $\mathrm{P}_{72}(x, y)=\left(\frac{1}{3}+\frac{x}{3}, \frac{1}{3}-\frac{x}{3}+\frac{y}{3}\right)$ & if $y \leq 2 x-1$ and $1 / 2 \leq x \leq 2 / 3$ \\
\hline & $\mathrm{P}_{73}(x, y)=\left(\frac{1}{3}+\frac{x}{3}, \frac{1}{2}+\frac{y}{2}\right)$ & $\begin{array}{l}\text { if } y \geq 1 / 2-x / 2 \text { and } 1 / 3 \leq x \leq 1 / 2 \text { or } \\
\text { if } y \geq 2 x-1 \text { and } 1 / 2 \leq x \leq 2 / 3\end{array}$ \\
\hline
\end{tabular}

\section{Appendix B: Maps for the Vicsek Fractal}

The Vicsek fractal is constructed using the five affine contraction maps presented in Table B1 and the corresponding five contraction maps found in Table B2 are required for the creation of the corresponding tessellation. As in Section A1 the coordinate functions in these tables are dimensionless.

Table B1 Contraction maps for the Vicsek fractal [21].

\begin{tabular}{|l|l|}
\hline $\mathrm{S}_{1}(x, y)=\left(\frac{x}{3}, \frac{1}{3}+\frac{y}{3}\right)$ & $\mathrm{S}_{2}(x, y)=\left(\frac{1}{3}+\frac{x}{3}, \frac{1}{3}+\frac{y}{3}\right)$ \\
\hline $\mathrm{S}_{3}(x, y)=\left(\frac{1}{3}+\frac{x}{3}, \frac{y}{3}\right)$ & $\mathrm{S}_{4}(x, y)=\left(\frac{2}{3}+\frac{x}{3}, \frac{1}{3}+\frac{y}{3}\right)$ \\
\hline $\mathrm{S}_{5}(x, y)=\left(\frac{1}{3}+\frac{x}{3}, \frac{2}{3}+\frac{y}{3}\right)$ \\
\hline
\end{tabular}


Table B2. Contraction maps for the tessellation to mirror the Vicsek [21].

\begin{tabular}{|c|c|c|}
\hline \multirow{4}{*}{$\mathrm{P}_{1}(x, y)$} & $\mathrm{P}_{11}(x, y)=\left(\frac{x}{3},-\frac{x}{3}+\frac{2 y}{3}+\frac{1}{3}\right)$ & if $y \geq x$ and $y \geq-x+1$ \\
\hline & $\mathrm{P}_{12}(x, y)=\left(\frac{x}{3}, \frac{y}{3}+\frac{1}{3}\right)$ & if $y \leq x$ and $y \geq-x+1$ \\
\hline & $\mathrm{P}_{13}(x, y)=\left(\frac{x}{3}, \frac{x}{3}+\frac{2 y}{3}\right)$ & if $y \leq x$ and $y \leq-x+1$ \\
\hline & $\mathrm{P}_{14}(x, y)=\left(\frac{x}{3}, y\right)$ & if $y \geq x$ and $y \leq-x+1$ \\
\hline \multicolumn{3}{|c|}{ 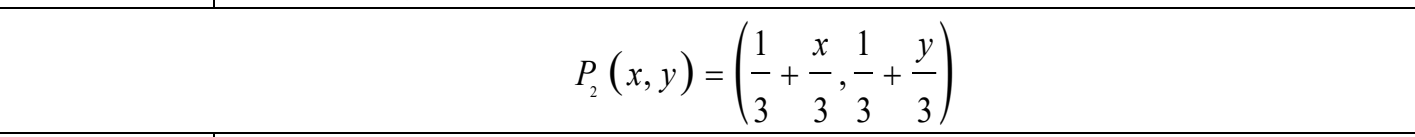 } \\
\hline \multirow{4}{*}{$\mathrm{P}_{3}(x, y)$} & $\mathrm{P}_{31}(x, y)=\left(\frac{x}{3}+\frac{1}{3}, \frac{y}{3}\right)$ & if $y \geq x$ and $y \geq-x+1$ \\
\hline & $\mathrm{P}_{32}(x, y)=\left(\frac{2 x}{3}-\frac{y}{3}+\frac{1}{3}, \frac{y}{3}\right)$ & if $y \leq x$ and $y \geq-x+1$ \\
\hline & $\mathrm{P}_{33}(x, y)=\left(x, \frac{y}{3}\right)$ & if $y \leq x$ and $y \leq-x+1$ \\
\hline & $\mathrm{P}_{34}(x, y)=\left(\frac{2 x}{3}+\frac{y}{3}, \frac{y}{3}\right)$ & if $y \geq x$ and $y \leq-x+1$ \\
\hline \multirow{4}{*}{$\mathrm{P}_{4}(x, y)$} & $\mathrm{P}_{41}(x, y)=\left(\frac{x}{3}+\frac{2}{3}, \frac{x}{3}+\frac{2 y}{3}\right)$ & if $y \geq x$ and $y \geq-x+1$ \\
\hline & $\mathrm{P}_{42}(x, y)=\left(\frac{x}{3}+\frac{2}{3}, y\right)$ & if $y \leq x$ and $y \geq-x+1$ \\
\hline & $\mathrm{P}_{43}(x, y)=\left(\frac{x}{3}+\frac{2}{3},-\frac{x}{3}+\frac{2 y}{3}+\frac{1}{3}\right)$ & if $y \leq x$ and $y \leq-x+1$ \\
\hline & $\mathrm{P}_{44}(x, y)=\left(\frac{x}{3}+\frac{2}{3}, \frac{y}{3}+\frac{1}{3}\right)$ & if $y \geq x$ and $y \leq-x+1$ \\
\hline $\mathrm{P}_{5}(x, y)$ & $\mathrm{P}_{51}(x, y)=\left(x, \frac{y}{3}+\frac{2}{3}\right)$ & if $y \geq x$ and $y \geq-x+1$ \\
\hline
\end{tabular}




\begin{tabular}{|ll|}
$\mathrm{P}_{52}(x, y)=\left(\frac{2 x}{3}+\frac{y}{3}, \frac{y}{3}+\frac{2}{3}\right)$ & if $y \leq x$ and $y \geq-x+1$ \\
\hline $\mathrm{P}_{53}(x, y)=\left(\frac{x}{3}+\frac{1}{3}, \frac{y}{3}+\frac{2}{3}\right)$ & if $y \leq x$ and $y \leq-x+1$ \\
\hline $\mathrm{P}_{54}(x, y)=\left(\frac{2 x}{3}-\frac{y}{3}+\frac{1}{3}, \frac{y}{3}+\frac{2}{3}\right)$ & if $y \geq x$ and $y \leq-x+1$ \\
\hline
\end{tabular}

\section{Acknowledgements}

The authors would like to acknowledge the Higher Committee for Education Development in Iraq for providing support for Zainab Sedqi to facilitate her doctoral research at the University of Manchester. 


\section{References}

[1] B. Mandelbrot, The fractal geometry of nature. 1977. W. H. Freeman and company.

[2] L. Gardi, The Mandelbrot set and the fractal nature of light, the Universe, and everything. 8832, 10.1117/12.2023739,SPIE, 2013.

[3] A.J. Salim and J.K. Ali. Design of internal dual-band printed monopole antenna based on Peano-type fractal geometry for WLAN USB dongle application. In Progress in Electromagnetics Research Symposium Proceedings. China, 2011. 12-16.

[4] T. Ozaki, and M. Nishigori, Crystal thickness dependence of pre-fractal domain structures in triglycine sulfate. Journal of the Korean Physical Society, 1988. 32(3).p.780-782,1998.

[5] M.G. Blyth, and C. Pozrikidis, Heat conduction across irregular and fractal-like surfaces. International Journal of Heat and Mass Transfer, 2003. 46: p. 13291339.

[6] S. Castineira-Ibanez, D. Tarrazo-Serrano, C. Rubio, P. Candelas and A. Uris, An ultrasonic lens design based on pre-fractal structures. Symmetry, 2016. 8(28): p.7,

[7] V.E. Tarasov, Fractional hydrodynamic equations for fractal media. Annals of Physics, 2005. 318: p. 286-307.

[8] S. Butera, and M. D. Paola, A physically based connection between fractional calculus and fractal geometry. Annals of Physics, 2014. 350: p. 146-158.

[9] Z. Hao and W. Qilin, Application study of fractal theory in mechanical transmission. Chinese Journal of Mechanical Engineering, 2016. 29(5): p. 871879.

[10] M. F. Bransley, Fractals Everywhere. Academia Press, Inc. 1993.

[11] A. Carpinteri, N. Pugno and A. Sapora, Free vibration analysis of a von Koch beam. International Journal of Solids and Structures, 2010. 47(11): p. 15551562.

[12] J. H. Jeng, V. V. Varadan and V.K. Varadan, Fractal finite element mesh generation for vibration problems. The Journal of the Acoustical Society of America, 1987. 82.

[13] T. Lewinski, Plates, Laminates and Shells: asymptotic analysis and homogenization 2000. London, ISBN: 978-981-281-369-5, World Scientific.

[14] O. Ram, G. Ben-Dor, and O. Sadot, On the pressure build up behind an array of perforated plates impinged by a normal shock wave. Experimental Thermal and Fluid Science, 2018. 92: p. 211-221. 
[15] J. Wang, P. Rubini, and Q. Qin, Application of a porous media model for the acoustic damping of perforated plate absorbers. Applied Acoustics, 2017. 127: p. 324-335.

[16] A. Efendioglu, J. Mendez, and H. Turkoglu, The numerical analysis of the flow and separation efficiency of a two-phase horizontal oil-gas separator with an inlet diverter and perforated plates Advances in Fluid Mechanics, 2014. 82: p. 133-142.

[17] F.J. Taguas, M.A. Martin, and E. Perfect, Simulation and testing of self-similar structures for soil particle-size distributions using iterated function systems. Geoderma, 1998. 88: p. 191-203.

[18] A.L. Kalamkarov, I.V. Andrianov, and V.V. Danishevs'kyy, Asymptotic homogenization of composite materials and structures. Applied Mechanics, 2009. 62(3):030802-030802-20. doi:10.1115/1.3090830

[19] A.A. Pan'kov, Generalized self-consistent method: modelling and computation of effective elastic properties of composites with composite or hollow inclusions. Mechanics of Composite Material, 1998. 34(2):p. 123-131.

[20] K. Davey, and R. Prosser, Analytical solutions for heat transfer on fractal and pre-fractal domains. Applied Mathematical Modelling, 2013. 37: p. 554-569.

[21] K. Davey, R. Prosser, and C. Jiang, Heat transfer through fractal-like porous media: A tessellated continuum approach. Computers \& Structures, 2015. 151: p. $58-72$.

[22] C. Jiang, K. Davey, and R. Prosser, A tessellated continuum approach to thermal analysis: discontinuity networks. Continuum Mechanics and Thermodynamics, 2017. 29(1): p. 145-186.

[23] K. Davey, C. Jiang, and R. Prosser, Tessellated continuum mechanics: A Galerkin finite element method. Computers \& Structures, 2016. p,157-183.

[24] K. Davey, R. Darvizeh, and A. Al-Tamimi, Scaled metal forming experiments: A transport equation approach. International Journal of Solids and Structures 2017. 125: p. 184-205.

[25] A. Al-Tamimi, R. Darvizeh, and K. Davey, Scaling of metal forming processes. Procedia Engineering, 2017. 207: p. 1069-1074.

[26] R. Ochoa-Cabrero, T. Alonso-Rasgado, and K. Davey, Scaling in biomechanical experimentation: a finite similitude approach. Journal of The Royal Society Interface, 2018. 15(143).

[27] M. Moghaddam, K. Davey, R. Darvizeh and A. Darvizeh, Scaling of the powder compaction process. International Journal of Solids and Structures, 2018. 144-145: p. 192-212.

[28] B. Zohuri, Dimensional analysis and self-similarity methods for engineers and scientists. ISBN:9783-319-13475-8, Springer, 2015. 
[29] Y.Ichikawa, and A.P.S. Selvadurai, Transport phenomena in porous media. Springer, Berlin, Heidelberg, 2012.

[30] W. Flügge, Tensor Analysis and Continuum Mechanics. , Springer-Verlag Berlin Heidelberg GmH, 1972.

[31] M. Smith, ABAQUS/Standard User's Manual, Version 6.14, Simulia, 2014. 
Table 1. Properties and dimensions of tessellated beams under isotropic scaling.

\begin{tabular}{|c|c|c|c|c|}
\hline \multicolumn{2}{|c|}{$k$} & 1 & 2 & 3 \\
\hline \multirow{3}{*}{ 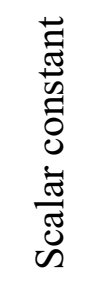 } & $\alpha^{\rho}$ & 1.0 & 1.0 & 1.0 \\
\hline & $\alpha^{u}$ & 1.5 & 2.25 & 3.375 \\
\hline & $\alpha^{v}$ & 1.5 & 2.25 & 3.375 \\
\hline \multirow{3}{*}{ 㤩 } & $L_{p s}(\mathrm{~m})$ & 0.3333 & 0.1111 & 0.0370 \\
\hline & $\rho_{p s}\left(\mathrm{~kg} / \mathrm{m}^{3}\right)$ & 1.0 & 1.0 & 1.0 \\
\hline & $E_{p s}\left(\mathrm{~N} / \mathrm{m}^{2}\right)$ & 1.0 & 1.0 & 1.0 \\
\hline \multirow{3}{*}{ 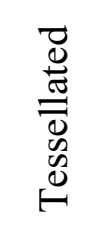 } & $L_{t s}(\mathrm{~m})$ & 0.5 & 0.2500 & 0.1250 \\
\hline & $\rho_{t s}\left(\mathrm{~kg} / \mathrm{m}^{3}\right)$ & 0.2700 & 0.0878 & 0.0026 \\
\hline & $E_{11}\left(\mathrm{~N} / \mathrm{m}^{2}\right)$ & 0.666 & 0.4444 & 0.2963 \\
\hline
\end{tabular}


Table 2. Properties and dimensions of tessellated beams under anisotropic scaling.

\begin{tabular}{|c|c|c|c|}
\hline \multicolumn{2}{|c|}{ Properties } & Case 1 & Case 2 \\
\hline \multirow{3}{*}{ 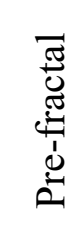 } & $L_{p s}(\mathrm{~m})$ & 0.037 & 0.037 \\
\hline & $\rho_{p s}\left(\mathrm{~kg} / \mathrm{m}^{3}\right)$ & 1.0 & 1.0 \\
\hline & $E_{p s}\left(\mathrm{~N} / \mathrm{m}^{2}\right)$ & 1.0 & 1.0 \\
\hline \multirow{3}{*}{ 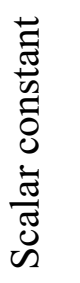 } & $\alpha^{\rho}$ & 1.0 & 1.0 \\
\hline & $\alpha^{u}$ & 3.375 & 11.39063 \\
\hline & $\alpha^{v}$ & 3.375 & 11.39063 \\
\hline \multirow{10}{*}{ 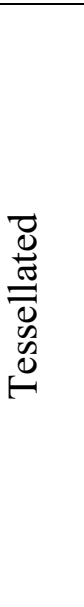 } & $L_{t s}(\mathrm{~m})$ & 0.125 & 0.125 \\
\hline & $W_{t s}(\mathrm{~m})$ & 0.02 & 0.037 \\
\hline & $T h_{t s}(\mathrm{~m})$ & 0.06750 & 0.228 \\
\hline & $\rho_{t s}\left(\mathrm{~kg} / \mathrm{m}^{3}\right)$ & 0.088 & 0.0142 \\
\hline & $E_{11}\left(\mathrm{~N} / \mathrm{m}^{2}\right)$ & 8.64976 & 18.076 \\
\hline & $E_{22}\left(\mathrm{~N} / \mathrm{m}^{2}\right)$ & 0.759375 & 1.789 \\
\hline & $E_{33}\left(\mathrm{~N} / \mathrm{m}^{2}\right)$ & 1.12 & 1.5 \\
\hline & $G_{11}\left(\mathrm{~N} / \mathrm{m}^{2}\right)$ & 1.28145 & 2.587 \\
\hline & $G_{12}\left(\mathrm{~N} / \mathrm{m}^{2}\right)$ & 49.2631 & 57.246 \\
\hline & $G_{23}\left(\mathrm{~N} / \mathrm{m}^{2}\right)$ & 14.5965 & 16.586 \\
\hline
\end{tabular}


Table 3. Number of elements on pre-fractal and tessellated plates.

\begin{tabular}{|c|c|c|c|}
\hline \multirow{2}{*}{ Structure Type } & \multicolumn{3}{|c|}{ Number of elements } \\
\cline { 2 - 4 } & $k=1$ & $k=2$ & $k=3$ \\
\hline Sierpinski carpet & 64 & 512 & 4096 \\
\hline Vicsek fractal & 40 & 200 & 1000 \\
\hline
\end{tabular}

Table 4. Properties and dimensions of the Sierpinski and Vicsek pre-fractals.

\begin{tabular}{|c|c|}
\hline Properties & Values \\
\hline Length $(\mathrm{m})$ & 0.9 \\
\hline Width $(\mathrm{m})$ & 0.9 \\
\hline Thickness $(\mathrm{m})$ & 0.01 \\
\hline Density $\left(\mathrm{kg} / \mathrm{m}^{3}\right)$ & 2698.8 \\
\hline Elasticity $\left(\mathrm{N} / \mathrm{m}^{2}\right)$ & $68.9 \times 10^{9}$ \\
\hline Poisson's ratio & 0.3 \\
\hline
\end{tabular}




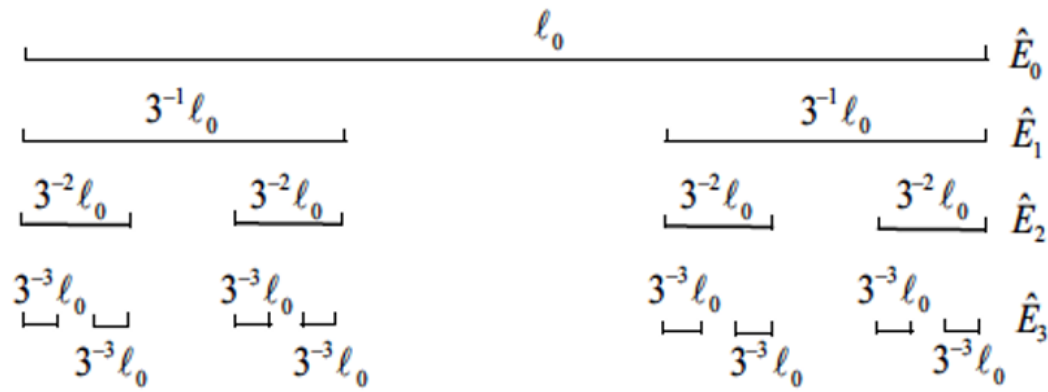

(a) Pre-fractal Cantor sets $\hat{E}_{k}$

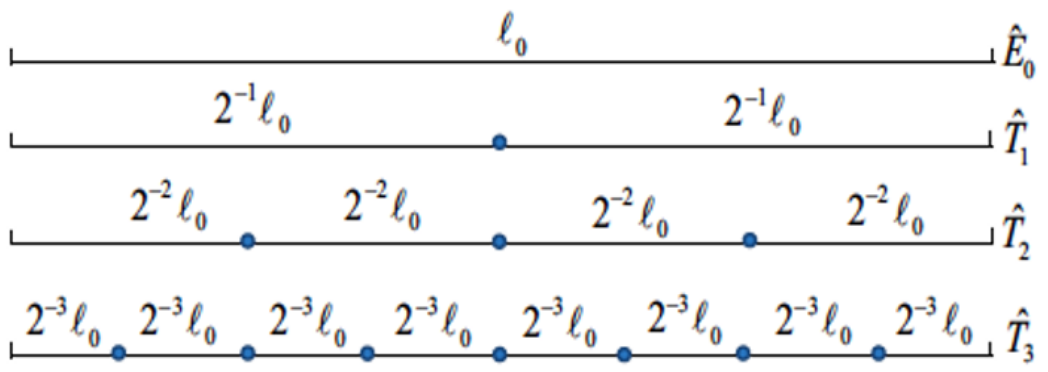

(b) Tessellated sets $\hat{T}_{k}$

Figure 1. Cantor dust pre-fractals and tessellations with discontinuity networks [22]. 


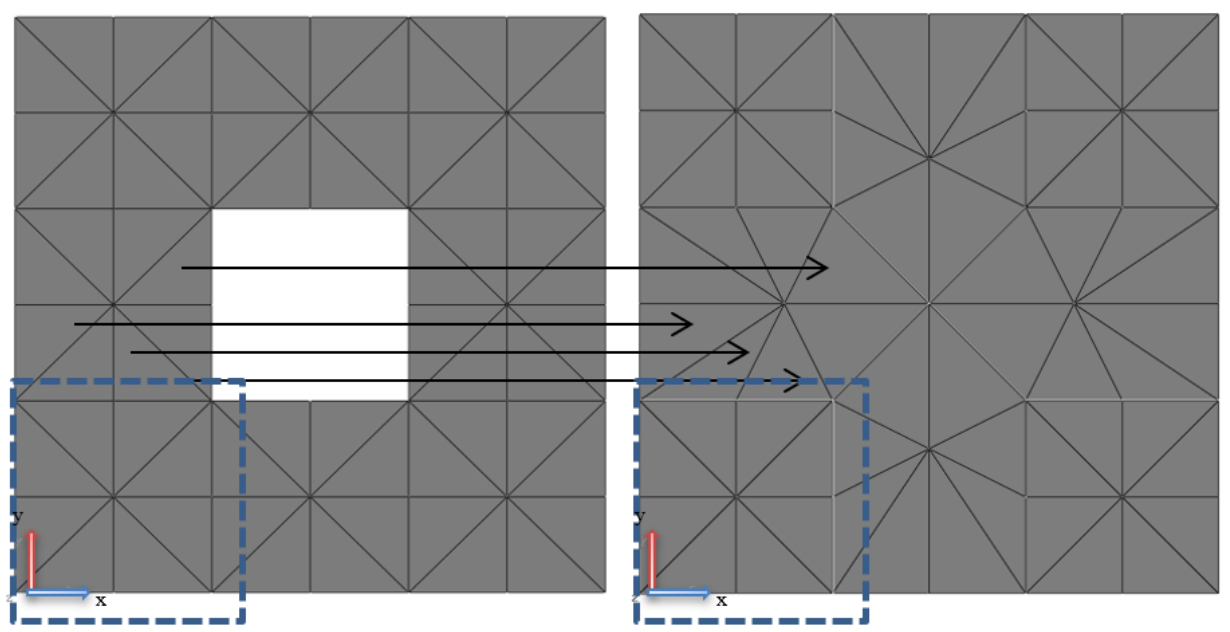

(a) $\hat{E}_{1}$

(b) $\hat{T}_{1}$

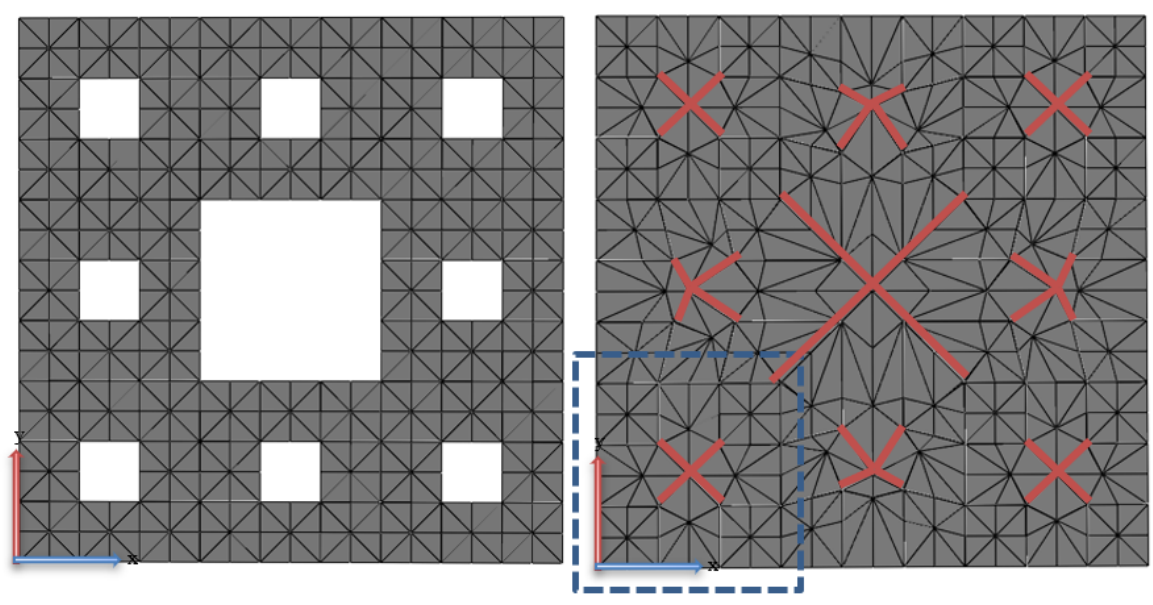
(c) $\hat{E}_{2}$
(d) $\hat{T}_{2}$ 


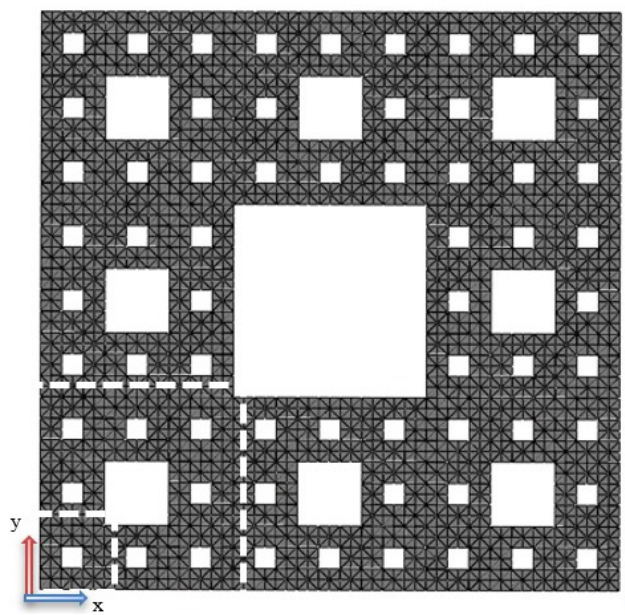

(e) $\hat{E}_{3}$

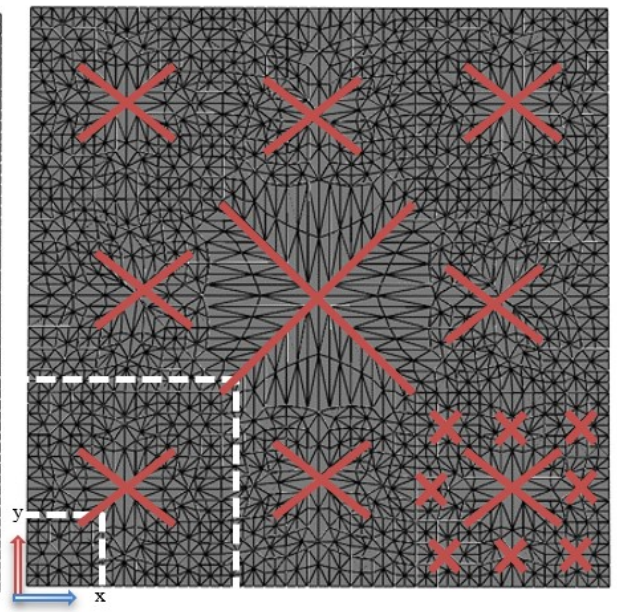

(f) $\hat{T}_{3}$

Figure 2. Sierpinski Carpet pre-fractals and tessellations with discontinuity networks.

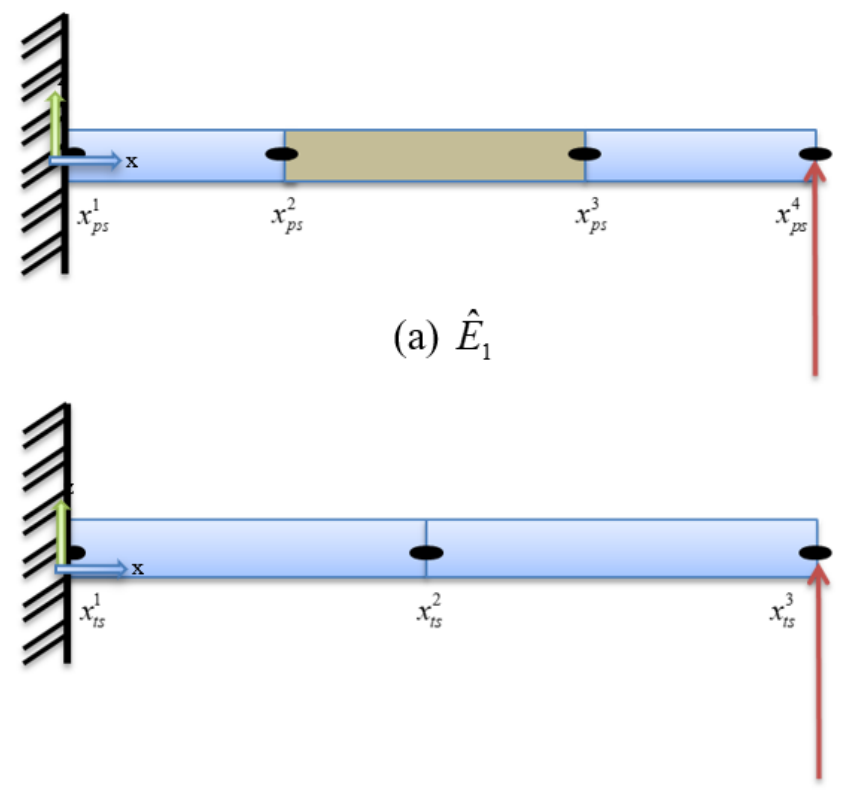

(b) $\hat{T}_{1}$

Figure 3. Pre-fractal and tessellated cantilever beams. 


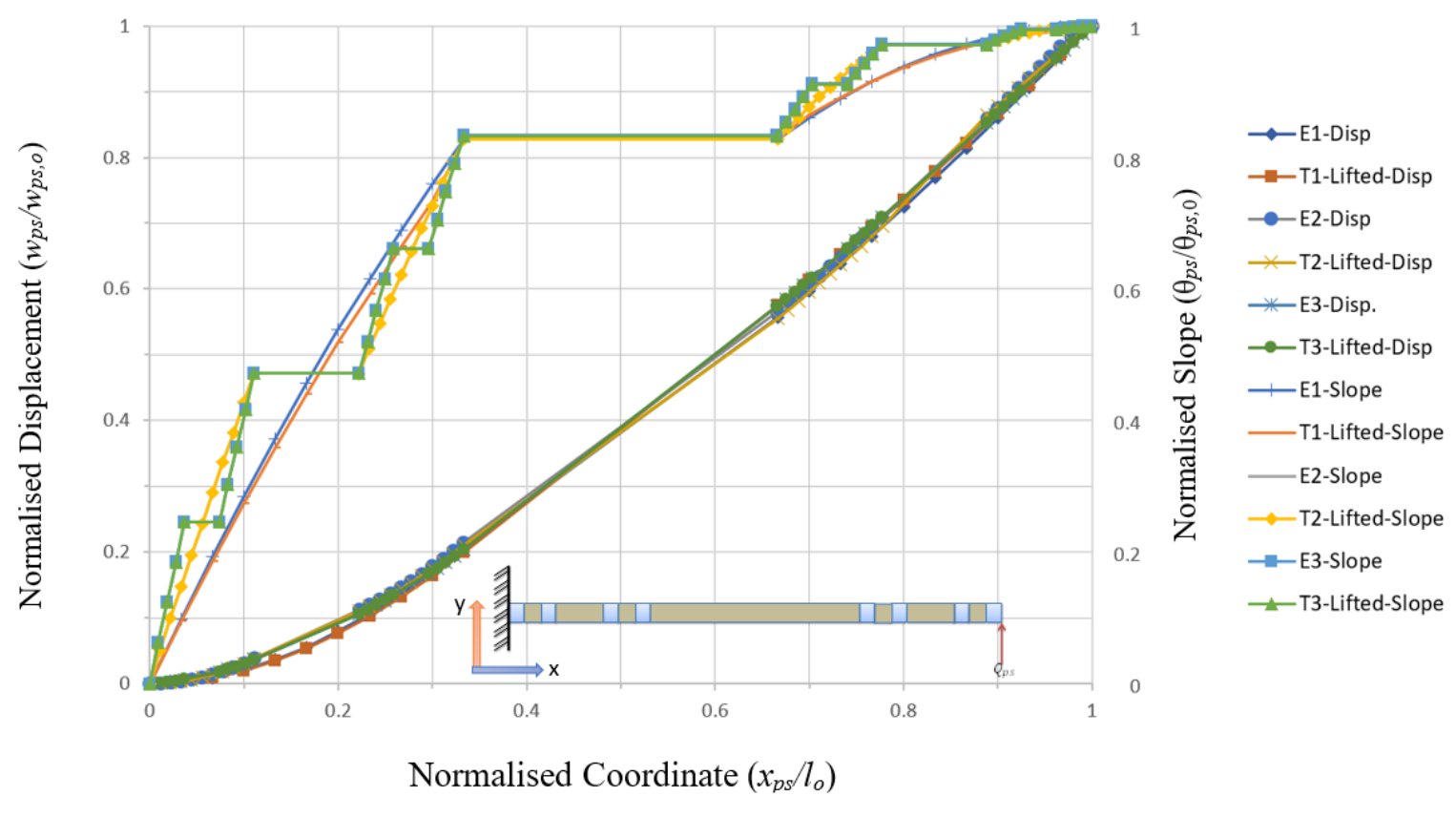

Figure 4. Analytical and FE predictions of deflection and slope on pre-fractal cantilevers.

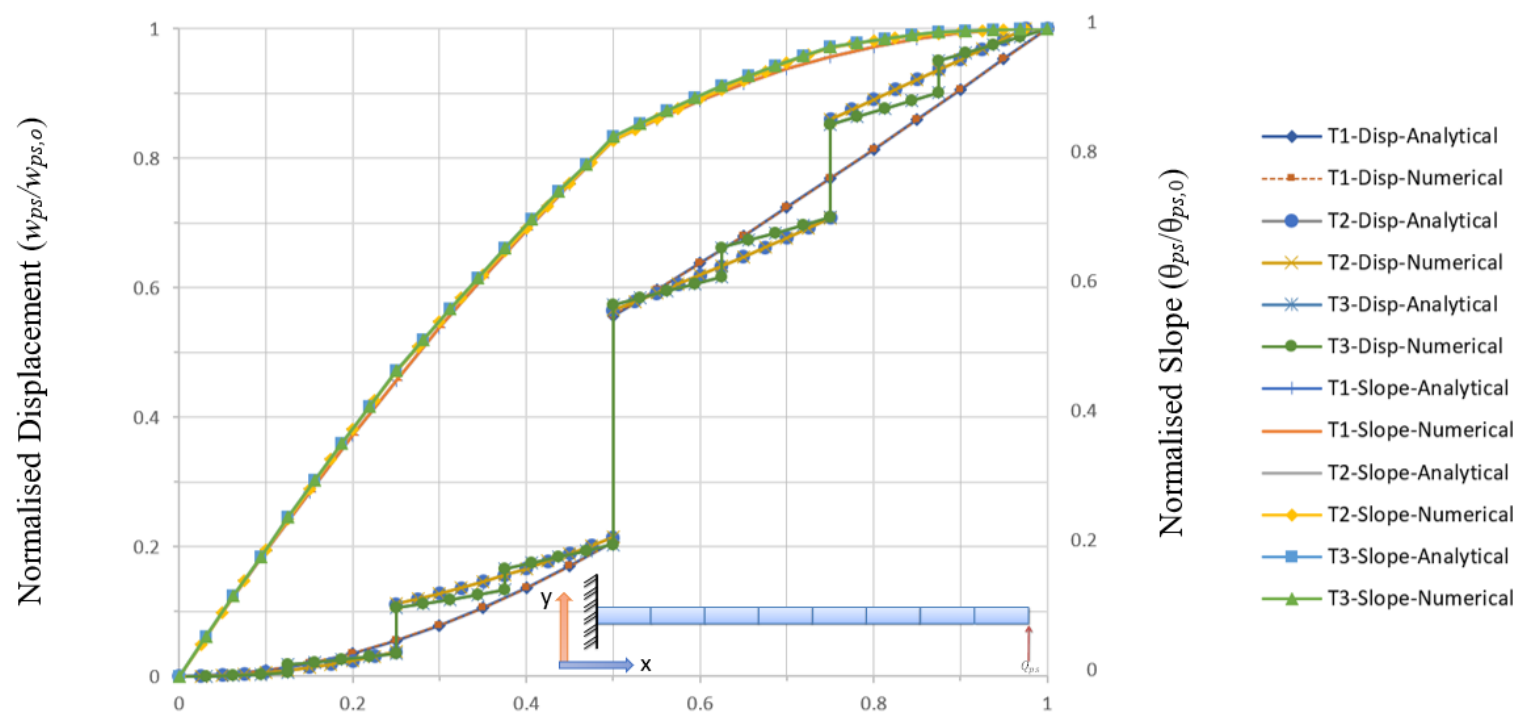

Normalised Coordinate $\left(x_{p s} / l_{o}\right)$

Figure 5. Analytical and FE predictions of deflection and slope on tessellated cantilevers. 


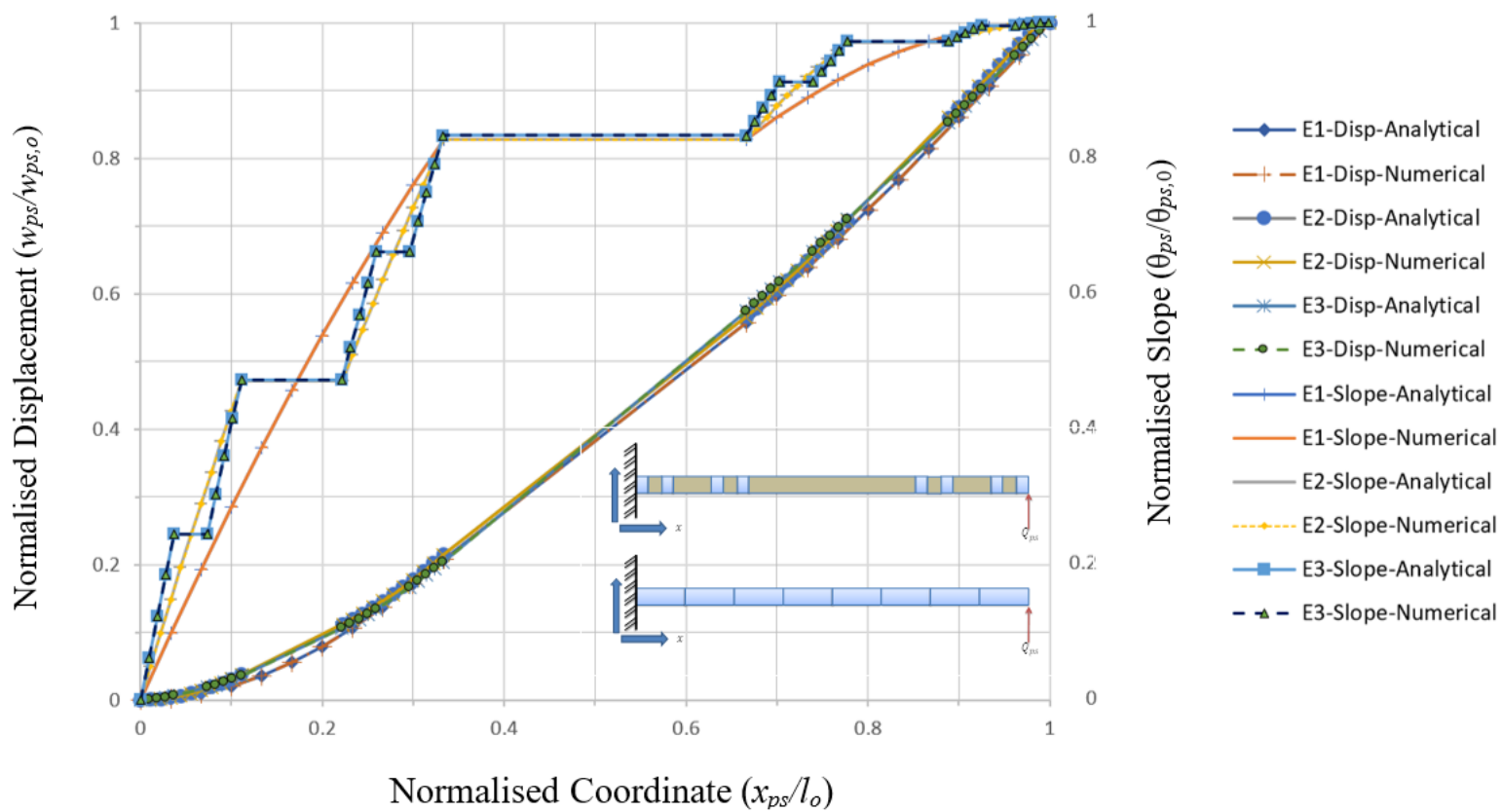

Figure 6. Direct-numerical and lifted-tessellated deflection and slope for cantilever prefractals.

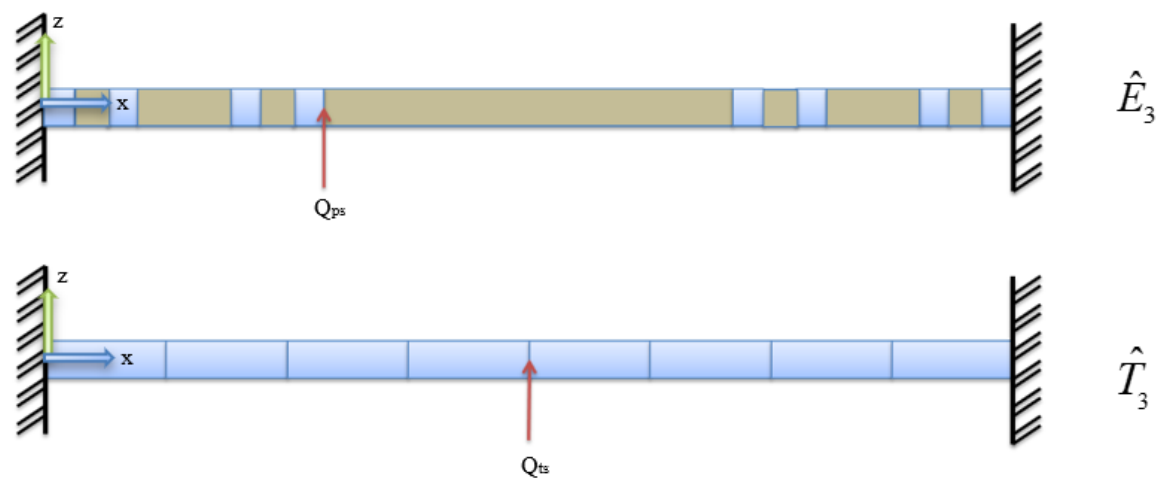

Figure 7. Pre-fractal and tessellated clamped (CC) beams. 


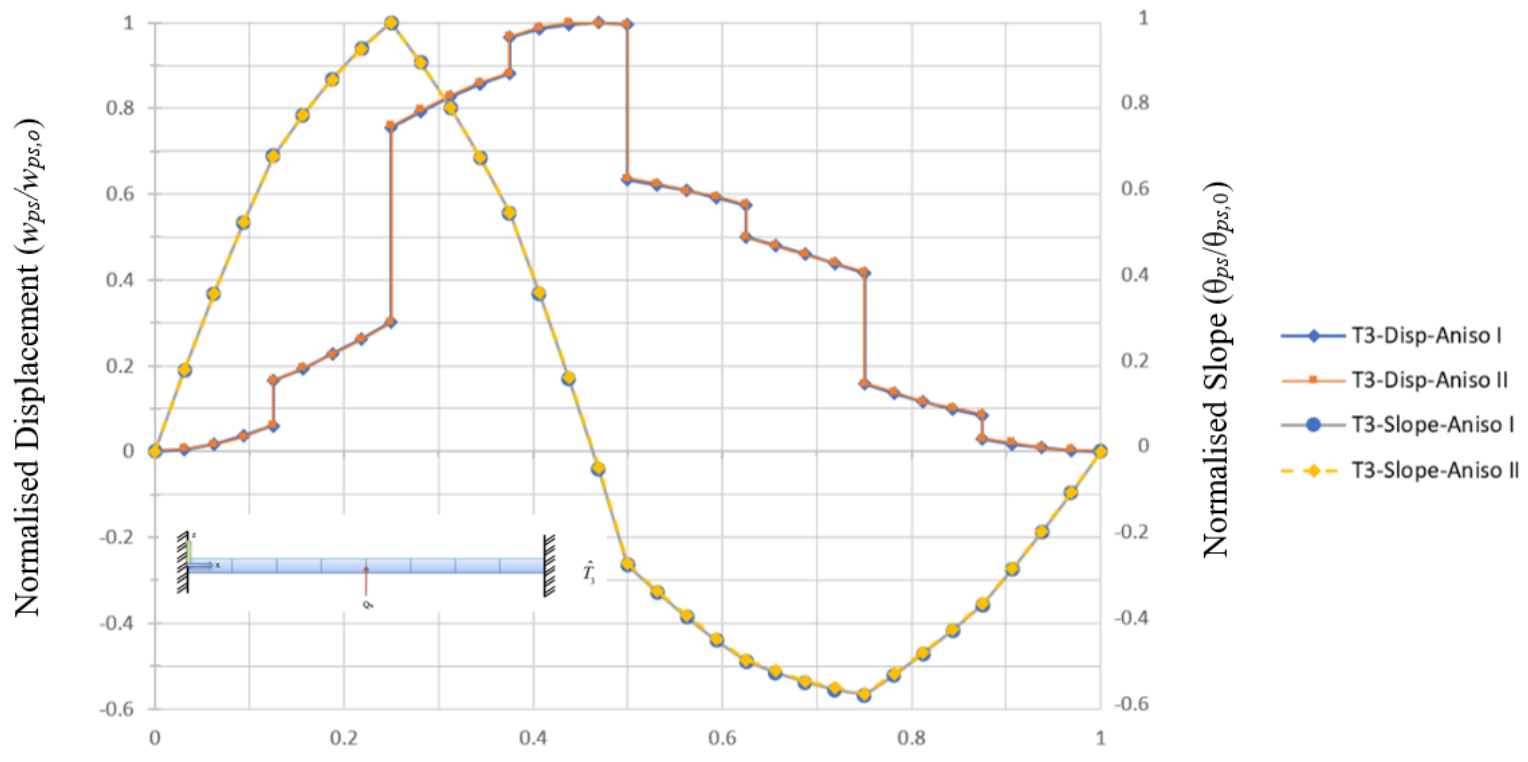

Normalised Coordinate $\left(x_{p s} / l_{o}\right)$

Figure 8 . FE prediction of deflection and slope on the tessellated encastre beam $\hat{T}_{3}$.

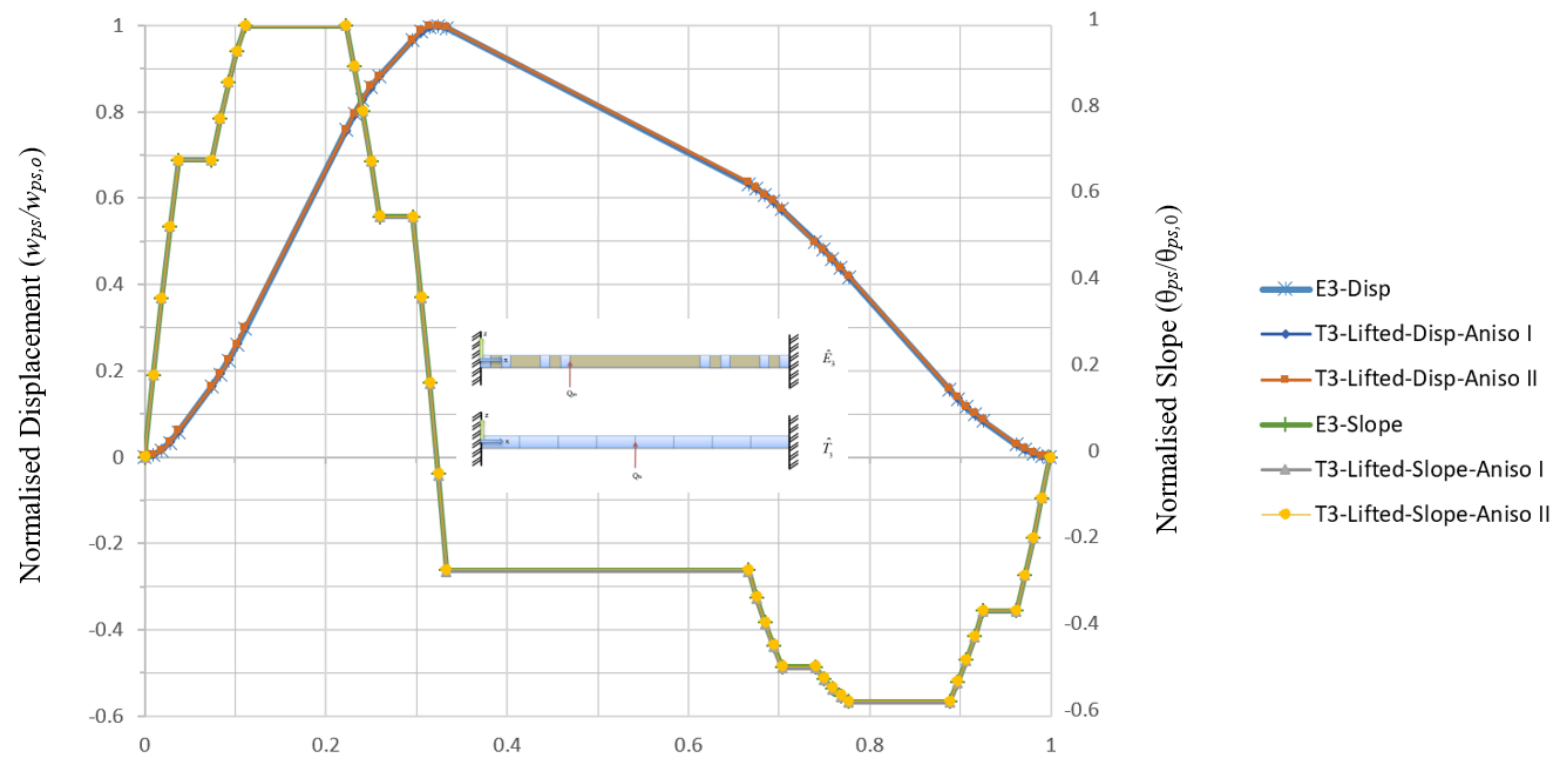

Normalised Coordinate $\left(x_{p s} l_{o}\right)$

Figure 9. Direct-numerical and lifted-tessellated deflection and slope for the encastre beam $\hat{E}_{3}$. 


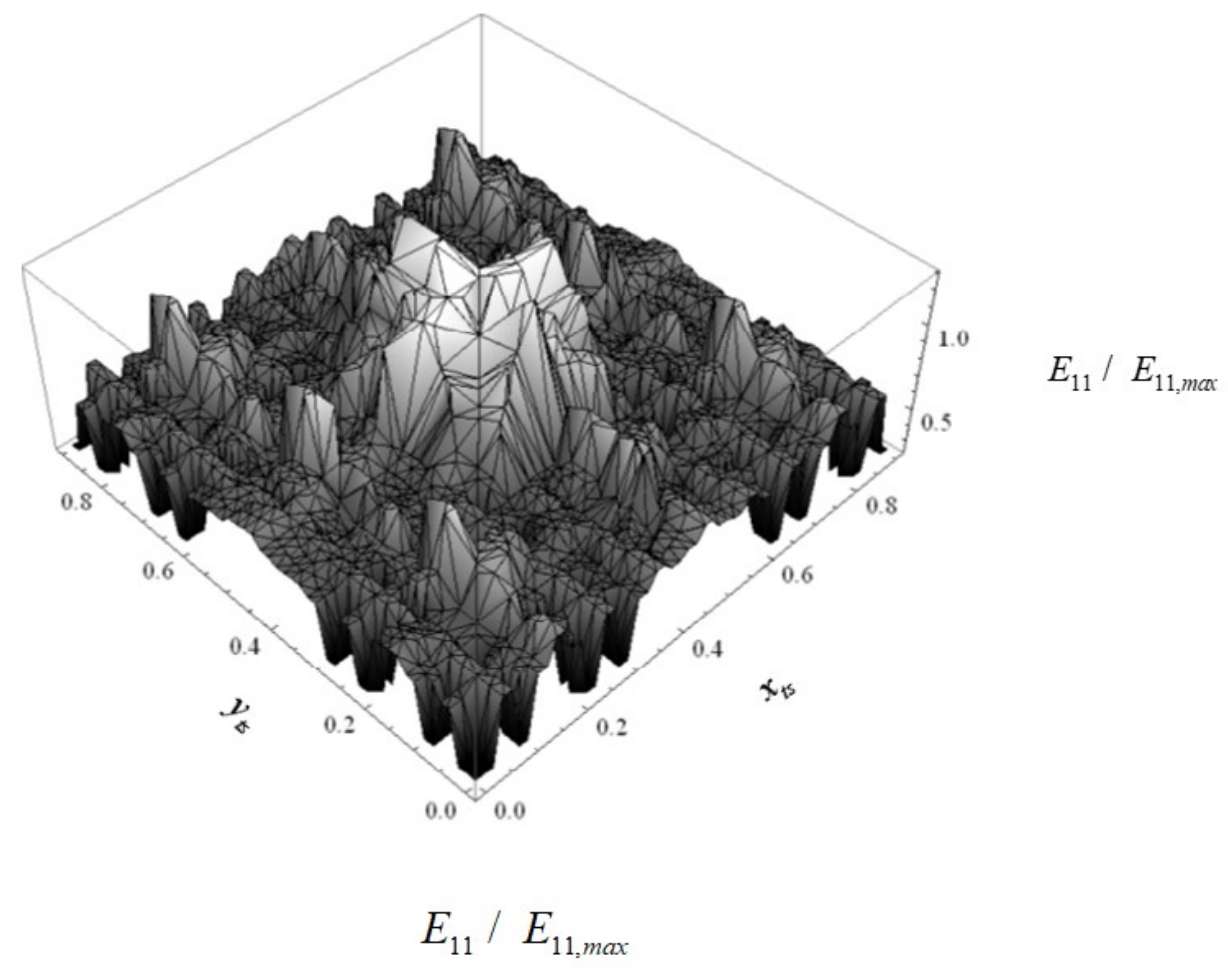

Figure 10. Normalised Young's moduli on Sierpinski carpet tessellation $\hat{T}_{3}$.

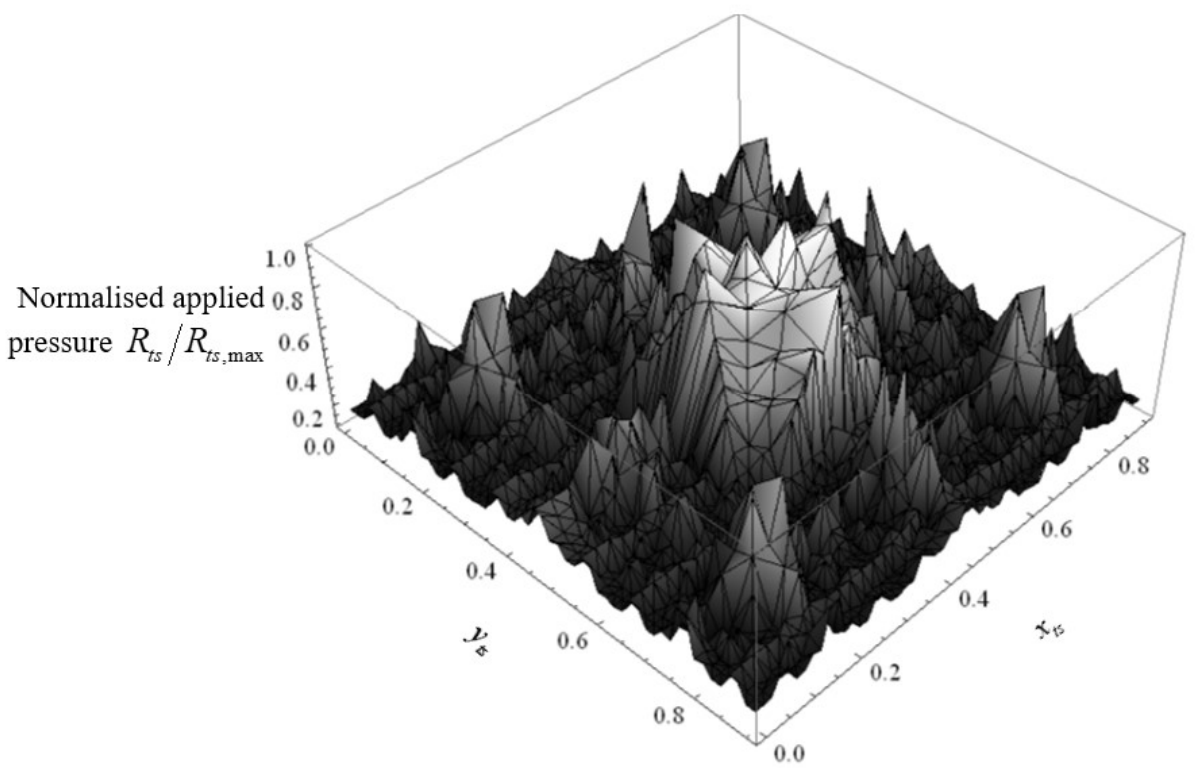

Figure 11. Normalised pressure distribution on Sierpinski carpet tessellation $\hat{T}_{3}$. 


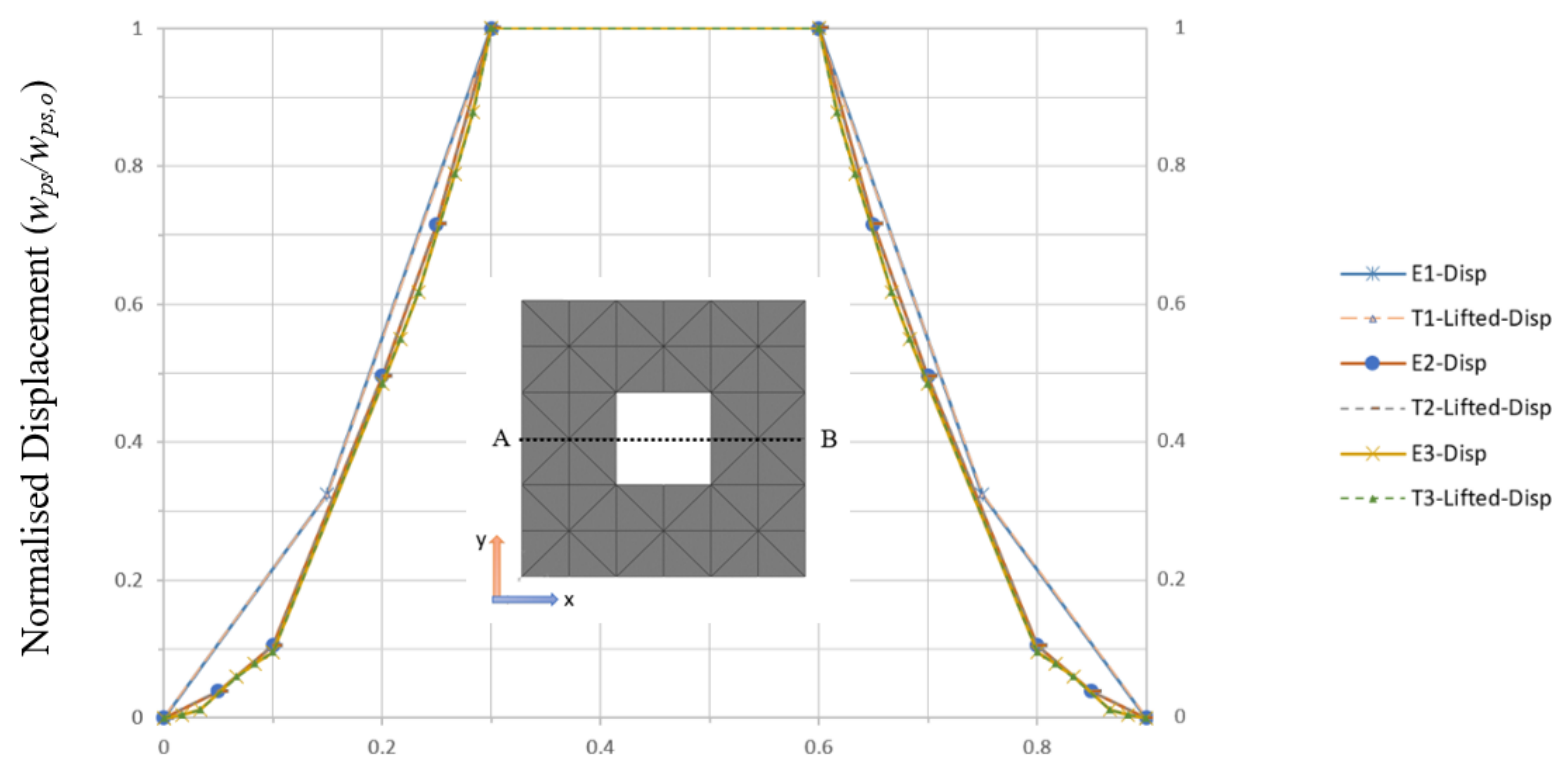

Normalised Coordinate $\left(x_{p s} / l_{o}\right)$

Figure 12. Direct-numerical and lifted-tessellated deflection for the pressure-loaded CCCC Sierpinski plates. 

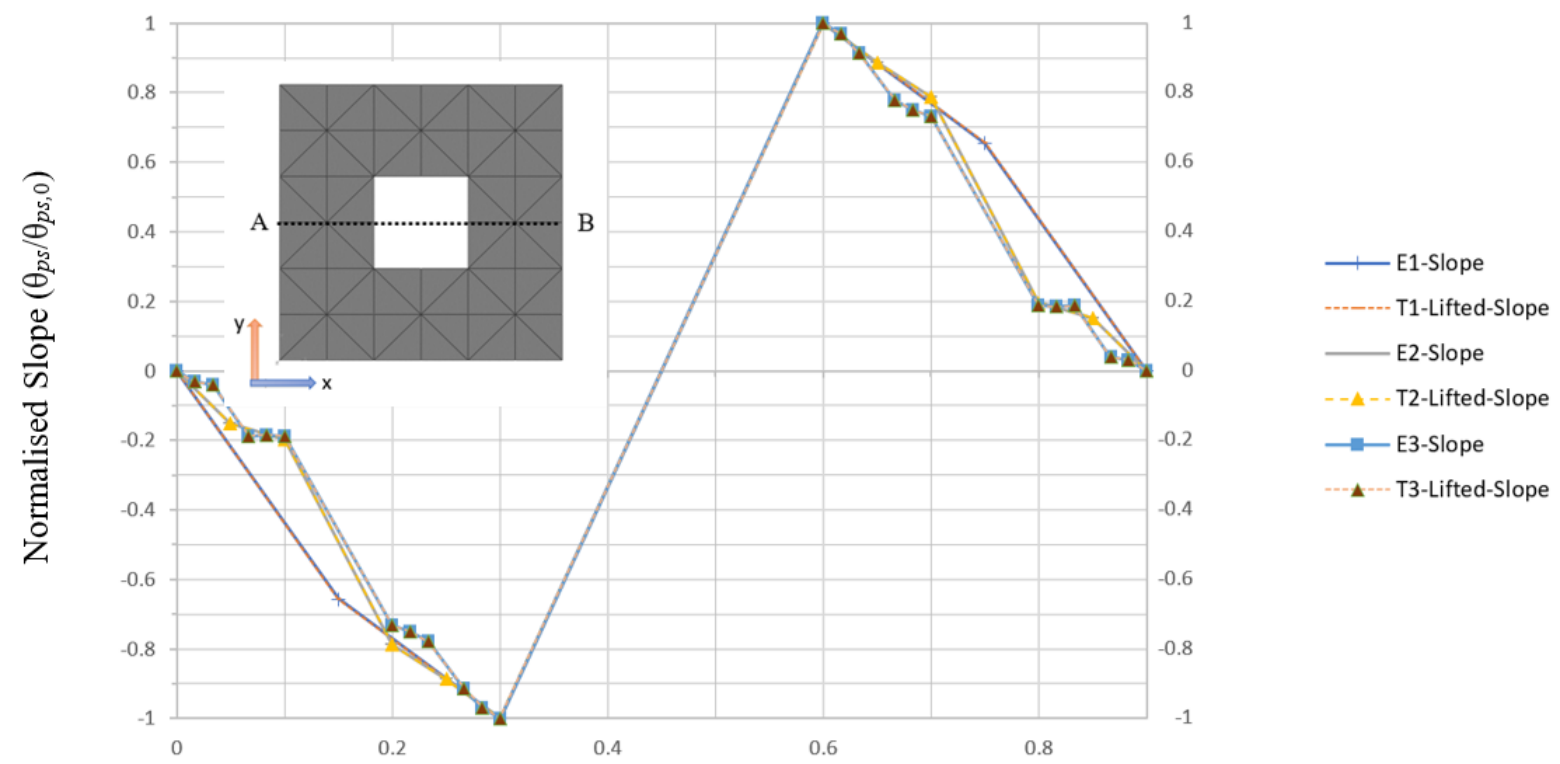

- - - - T2-Lifted-Slope

-E3-Slope

- T3-Lifted-Slope

Normalised Coordinate $\left(x_{p s} / l_{o}\right)$

Figure 13. Direct-numerical and lifted-tessellated slope $\left(\theta_{p s}=\partial w_{p s} / \partial x_{p s}\right)$ for the pressureloaded CCCC Sierpinski plates. 


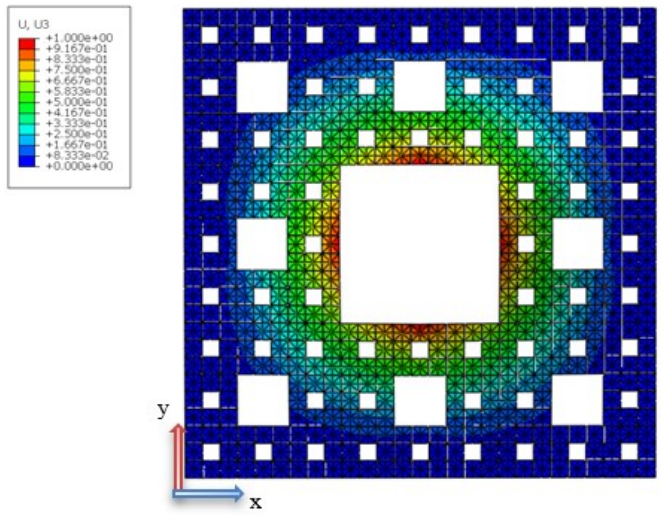

(a) Normalised lateral displacement $\left(w_{p s}\right)$ on $\hat{E}_{3}$
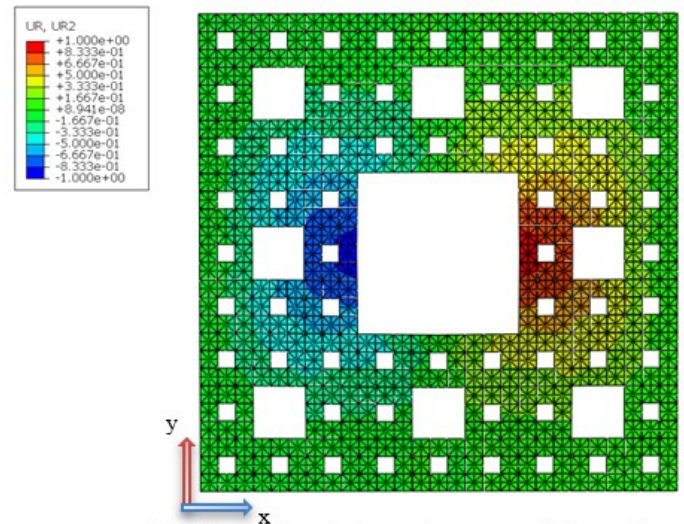

(c) Normalised slope $\left(\partial w_{p s} / \partial x_{p s}^{1}\right)$ on $\hat{E}_{3}$
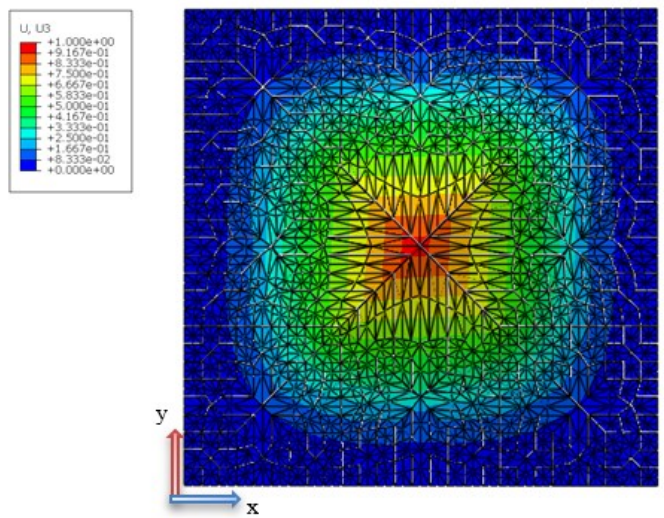

(b) Normalised lateral displacement $\left(w_{t}\right)$ on $\hat{T}_{3}$
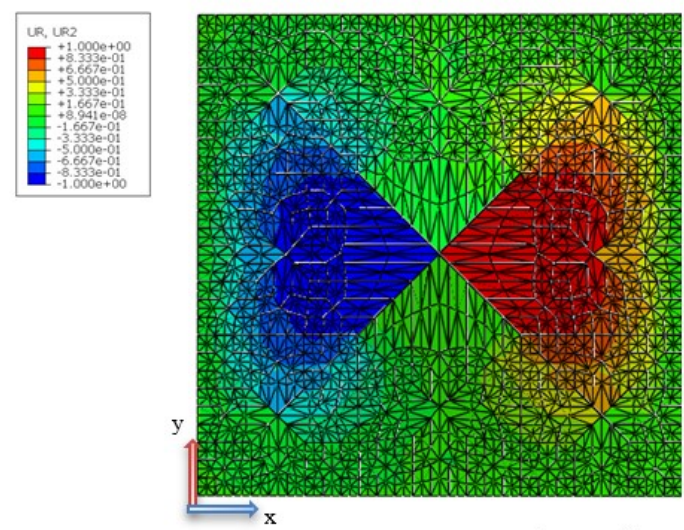

(d) Normalised Slope $\left(\partial w_{t s} / \partial x_{t s}^{1}\right)$ on $\hat{T}_{3}$

Figure 14. Overall response of pressure-loaded CCCC plates $\hat{E}_{3}$ and $\hat{T}_{3}$ for the Sierpinski carpet. 


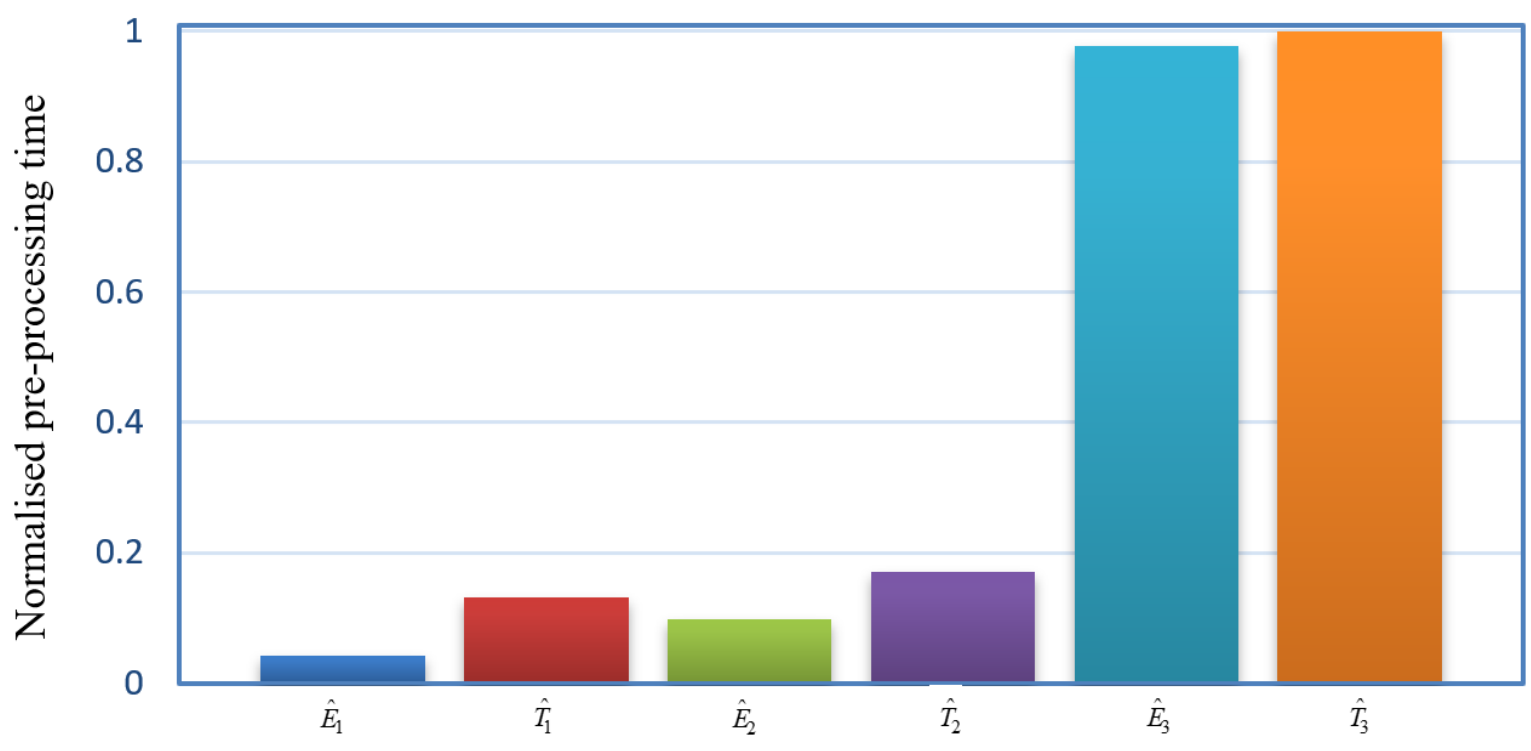

Figure 15. Normalised computational requirements for building pre-fractal and tessellation models.

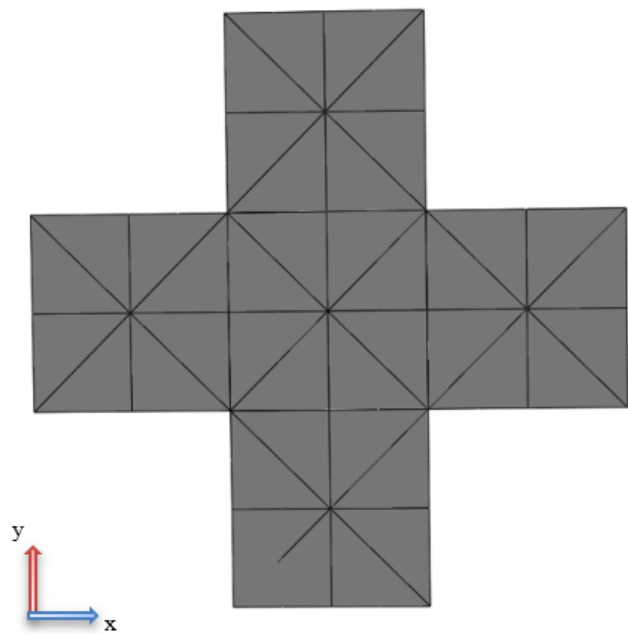

(a) $\hat{E}_{1}$

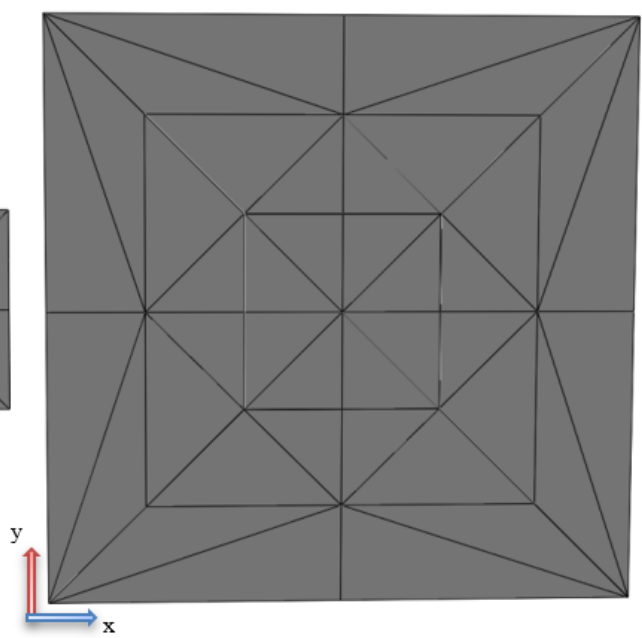

(b) $\hat{T}_{1}$ 


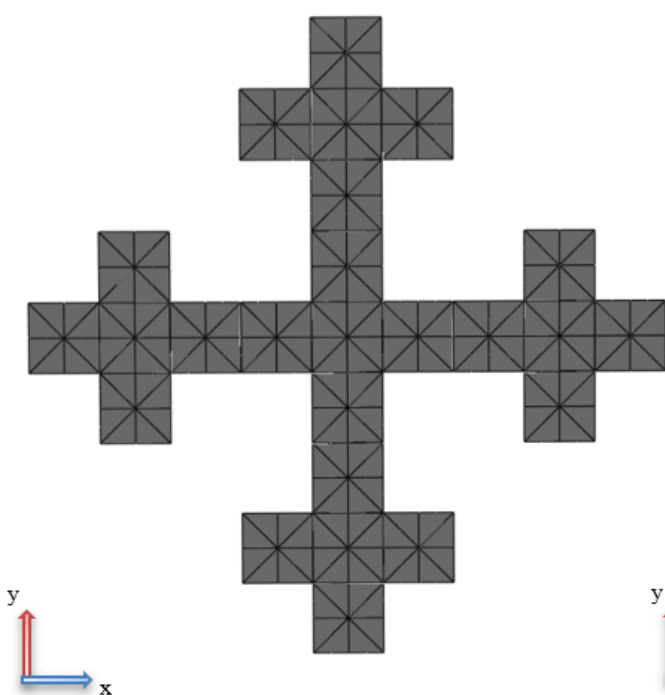

(c) $\hat{E}_{2}$

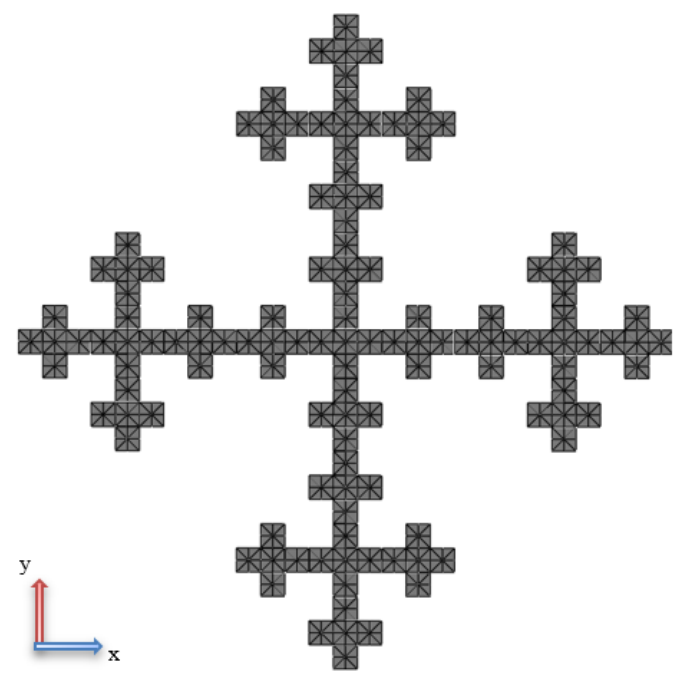

(e) $\hat{E}_{3}$

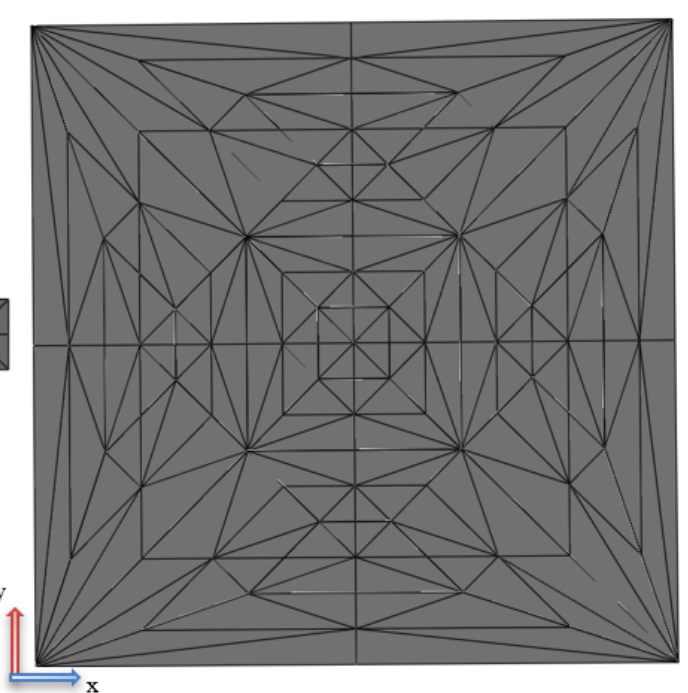

(d) $\hat{T}_{2}$

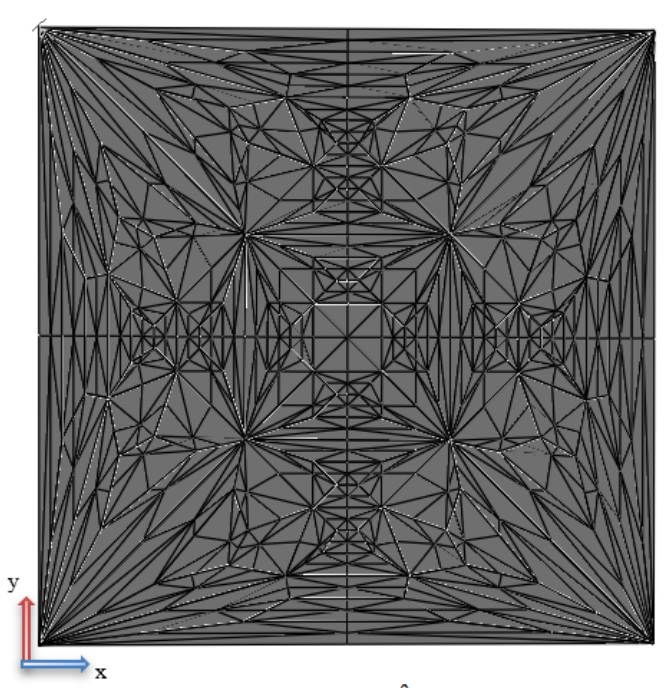

(f) $\hat{T}_{3}$

Figure 16. Mesh on Vicsek pre-fractals $\hat{E}_{k}$ and $\hat{T}_{k}(k=1: 3)$. 

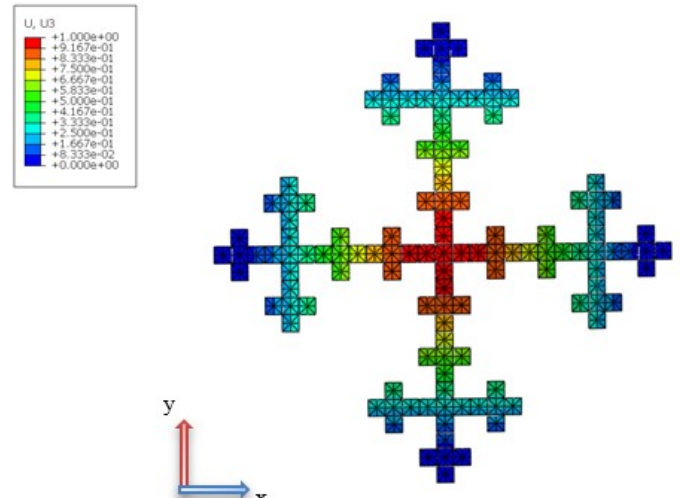

(a) Normalised lateral displacement $\left(w_{t}\right)$ on $\hat{E}_{3}$
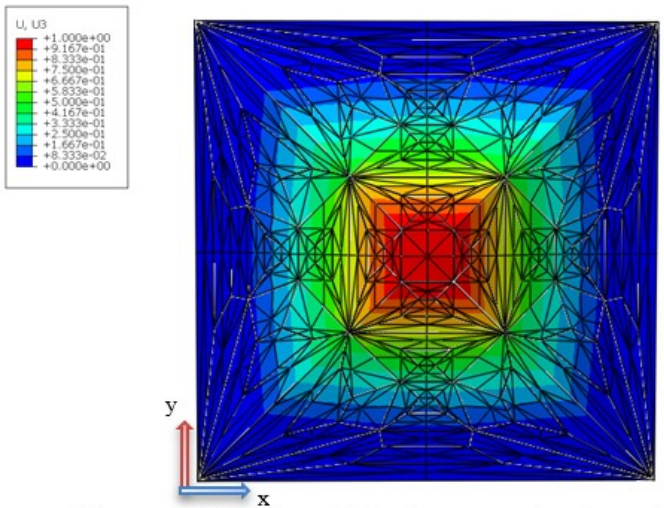

(b) Normalised lateral displacement $\left(w_{t 5}\right)$ on $\hat{T}_{3}$
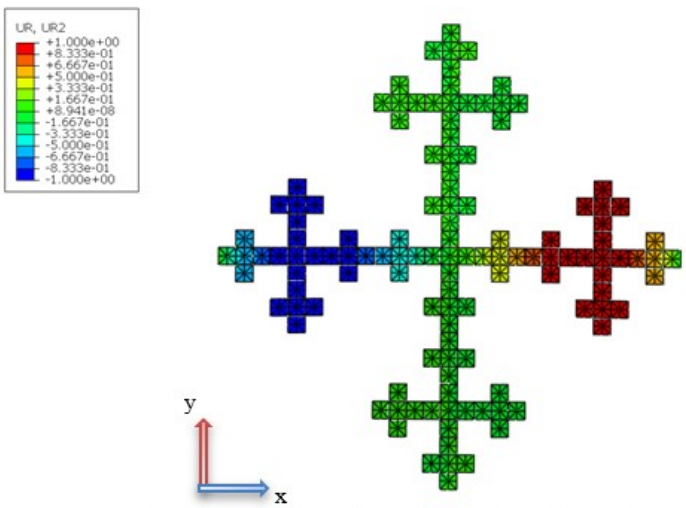

(c) Normalised slope $\left(\partial w_{p s} / \partial x_{p s}^{1}\right)$ on $\hat{E}_{3}$
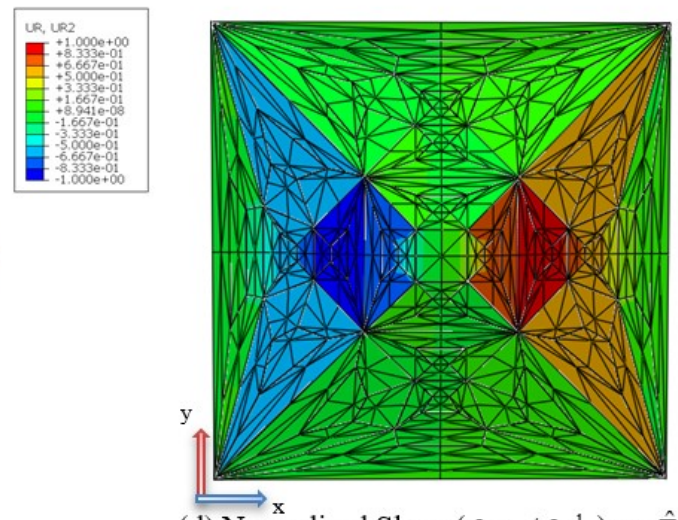

(d) Normalised Slope $\left(\partial w_{t s} / \partial x_{t s}^{1}\right)$ on $\hat{T}_{3}$

Figure 17. Overall response of centrally-loaded CCCC Vicsek plates $\hat{E}_{3}$ and $\hat{T}_{3}$. 


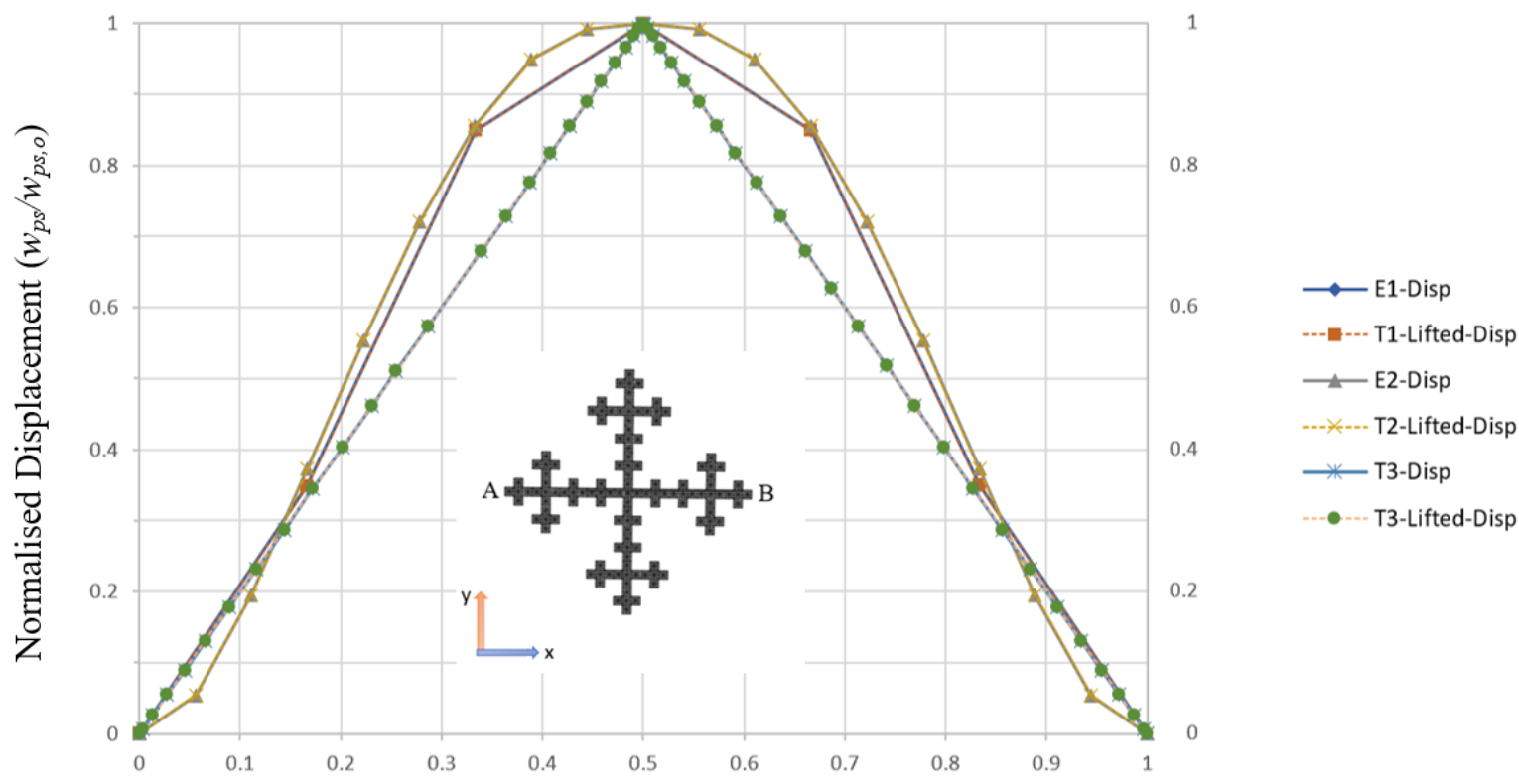

Normalised Coordinate $\left(x_{p s} / l_{o}\right)$

Figure 18. Displacement $\left(w_{p s}\right)$ on the centrally-loaded CCCC Vicsek plates.

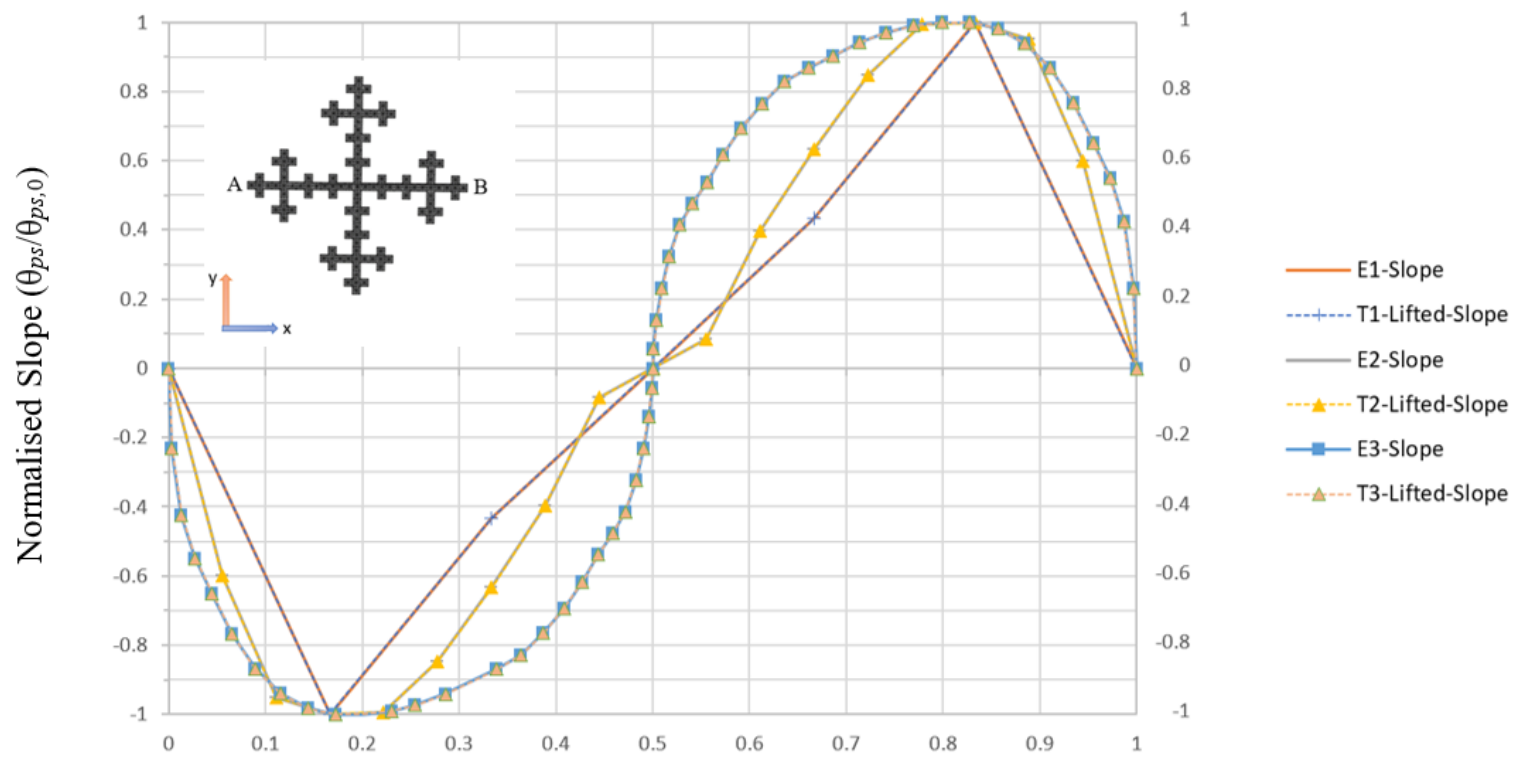

Normalised Coordinate $\left(x_{p s} / l_{o}\right)$

Figure 19. Slope $\left(\theta_{p s}=\partial w_{p s} / \partial x_{p s}\right)$ on the centrally-loaded CCCC Vicsek plates. 